\title{
Estructura organizativa e innovación en la Economía Social de Andalucía
}

\author{
Macarena Pérez-Suárez \\ Francisco Espasandín Bustelo \\ Isadora Sánchez-Torné
}

RESUMEN: Esta investigación persiguió dos objetivos: conocer la relación entre tres parámetros de diseño organizativo (centralización, formalización y preparación) y los tipos de innovación; y saber si existen configuraciones estructurales (arquetipos) en el sector de Economía Social, y en tal caso, determinar si diferentes configuraciones estructurales generan diferentes tipos de innovación. Para conseguir estos objetivos, se formularon seis hipótesis y se diseñó un modelo conceptual, utilizando el método inductivo a partir de varios análisis estadísticos (inferencias y análisis clúster) a una muestra de cooperativas y sociedades laborales en un límite territorial, Andalucía. Como resultado se identificó dos segmentos de empresas que innovan, probándose disparidad de clústers andaluces de Economía Social, y destacando el arquetipo de estructura orgánica y el alto número de innovaciones incrementales de proceso.

PALABRAS CLAVE: Arquetipos Estructurales, Innovación, Cooperativas, Análisis Clúster, Grupos Estratégicos.

CLAVES ECONLIT: M10, 031, P13.

Cómo citar este artículo / How to cite this article: PÉREZ-SUÁREZ, M., ESPASANDÍN, F. \& SÁNCHEZ-TORNÉ,

I. (2017): "Estructura organizativa e innovación en la economía social de Andalucía ", CIRIEC-España, Revista de Economía Pública, Social y Cooperativa, 90, 35-74.

Correspondencia: Macarena Pérez-Suárez, e-mail: mperez32@us.es; Francisco Espasandín Bustelo, e-mail: bustelo@us.es, e Isadora Sánchez-Torné, e-mail: isanchez6@us.es, Facultad de Ciencias Económicas y Empresariales de la Universidad de Sevilla, Avda. Ramón y Cajal, s/n, 41018 - Sevilla. 


\section{EXPANDED ABSTRACT}

\section{Organisational structure and innovation in the social economy of Andalusia}

Innovating companies improve their operation by increasing their level of flexibility and by adapting to the needs of their stakeholders. Innovation is also a key factor for business survival. Here, the system of Social Economy companies is observed.

Innovation is considered as the organisational capacity to develop processes and/or to produce and to commercialise a product and/or service in the market, by approaching a procedure that starts with an invention, and culminates in its adoption by the clients. Innovation is an intangible business asset derived from application, understanding, introduction, combination and transformation. Based on the literature, innovation is classified into various types: product, process, incremental and radical, among others. The results of innovation are not only conditioned by the organisation, because their determining or background factors are numerous.

In particular, in order to correct the weaknesses observed in the literature, and to provide the Directors of Social Economy companies with innovation management, the objectives of this study were twofold: first, to determine the relationship between the organisational design parameters (centralisation, formalisation, preparation and coordination) and the types of innovation; and second, to know if there are structural configurations in the Andalusian Social Economy sector, and if so, to determine if different structural configurations generate different types of innovation.

In this study, six hypotheses were formulated which constituted a conceptual model. The inductive method was used from several statistical analyses (inferences and cluster analysis) of a sample of cooperatives and worker-owned companies in Andalusia. Four hypotheses relate the innovation levels to the characteristics and types of organisational structures, whereas, the other two identify strategic groups within the innovating companies and the types of innovation.

The population under study was centred on the cooperative businesses and worker-owned companies that comprise the Andalusian Social Economy system. The collection of data was made in 2003, through a semi-structured questionnaire of ten questions. The extraction of the sample element (515 companies) was made by means of a simple random sampling procedure, based on chance and applied by means of tables of random numbers. Finally, for the statistical treatment of the collected data, the program SPSS/PC (V23) was used. 
Of the six formulated hypotheses, only one was rejected: "the centralisation of the organisational structure is greater in the Social Economy companies that do not innovate". The obtained results showed that the decentralisation levels are greater in the Social Economy companies which do not innovate. Next, the main results obtained through the five hypotheses that are supported are presented.

Firstly, it was verified that there is slightly less formalisation of behaviour in the Social Economy companies that innovate and, consequently, the structure is more organic. In addition, the levels of preparation of the senior management of the work teams, of the employees, and the level of coordination, is significantly less in non-innovating Social Economy companies. Therefore, the structural differences between the innovating and non-innovating Social Economy companies can be analysed through their level of decentralisation.

Secondly, two clusters could be identified which were composed of two strategic groups of innovating Social Economy companies, with different organisational structures. Specifically, cluster 1 was characterised by: a medium-to-high level of formalisation of behaviour, a medium-to-high level of preparation of the senior management, together with a medium level of preparation of the work teams and a moderate level of the employees, and a high degree of coordination and centralisation. Through these characteristics, except the high degree of centralisation, the companies that comprise this strategic group approximate to the mechanistic structure model.

Cluster 2 presents: a medium-to-high level of coordination and a low-to-medium level of formalisation, preparation of the senior management, preparation of the work teams, preparation of the employees, and centralisation. As opposed to the previous model, this strategic group approximates to an organic structure, through its low levels of formalisation of behaviour and, in spite of, its low degree of centralisation.

Finally, the innovating Social Economy companies of the two identified strategic groups resemble each other, because they obtained a high number of incremental process innovations. On the other hand, they are different, because proportionally, the companies whose structures approximate to the mechanist model, obtained more radical product innovations, and the companies which approximate to the organic structure model, obtained significantly more radical process innovations.

This study is not free of limitations, among them five limitations are highlighted: 1) the study provides a static view of the Andalusian Social Economy companies; 2) the sample is representative of the population, but it does not exclude problems of bias and heterogeneity, derived from the questionnaire being answered by the senior directors of the organisations, or the innovation managers; 3 ) The methods of multivariate analysis employed in themselves acquire their own conditions; 4) the problems of measurement of the "organisational structure" construct, and the consideration of only two criteria and four types of innovation; and, 5) the conceptual model only considers the organisational 
structure variable as a determinant of the types of innovation in Social Economy companies, when the determinants of the result of the innovation are really multiple.

The obtained results suggest proposing the following three groups: the Directors of the Social Economy companies which innovate, the Directors of the Social Economy companies that do not innovate, and the public administration.

From our understanding, the Directors of the Social Economy companies which innovate should consider the following suggestions: emphasise innovation more; understand that there is not one sole organisational structure to obtain different types of innovation; to the extent possible, incorporate resources, positions and bodies that stimulate innovation in the organisational structures; understand that companies can be innovating with any structure, and can cease to be so with any structure; modify the centralisation levels, for the sake of obtaining more organic organisational structures; work to create suitable tools to innovate; construct a common language of innovation and ensure that human resources policies are aligned with innovation; become aware that time is crucial to assess how the structure influences the innovation and financial results.

On the other hand, the Directors of Social Economy companies that do not innovate must be sensitive to the need to have suitable structures that facilitate the development and the performance of the capacities of product innovation, and to reduce formalisation levels.

Finally, the public administration must develop public policies that promote all types of innovation in the Social Economy companies, and not solely the radical innovations.

KEYWORDS: Structural Archetypes, Strategic Innovation, Cooperatives, Cluster Analyses, Groups. 


\section{1.- Introducción}

La importancia de la innovación es continua y sostenible, entre otros ámbitos, a nivel empresarial y académico. Desde el ámbito empresarial, las compañías innovadoras optimizan sus resultados económicos, sociales, y de mercado; además de mejorar su nivel de competitividad (López-Fernández, Serrano-Bedia y Gómez-López, 2011), ventaja competitiva (Hitt, Keats y Demarie, 1998; Hitt y Irlanda, 2000; Gudmundson, Tower y Hartman, 2003; Elenkov y Manev, 2005) y éxito (Tushrnan, Anderson y O'Reiuy, 1997). Entre los resultados económicos, la literatura consultada cita: la Reducción de Costes (Al Shaar, Khattab, Alkaied y Manna, 2015), Rentabilidad (Geroski, 1995; Johannessen, Olsen y Lumpkin, 2001), Productividad (McAdam y Keogh, 2004), Beneficio (López-Fernández et al., 2011), Rendimiento (Subramanian y Nilakanta, 1996; Gopalakrishnan, 2000; Zahra y Bogner, 2000; Guan y Ma, 2003; Wu y Zhuang, 2003; Zortea-Johnston, Darroch y Matear, 2012), Ventas y Desempeño (Quadros, Furtado, Bernardes y Franco, 2001). Asimismo en relación a los resultados de mercado, se transcriben los siguientes: Aumento de la cuota de mercado (McAdam y Keogh, 2004; López-Fernández et al., 2011), Aumento de la reputación (Gunday, Ulusoy, Kilica y Alpkan, 2011), Identificación de oportunidades de valor (Hitt, Hoskisson, Johnson y Moesel,1996; Rodríguez y Guzmán, 2013), Crecimiento (Amit y Zott, 2001; Wong et al., 2005; Lam, 2008; Menguc y Auh, 2010), Aumento de las exportaciones y Mejora de la calidad del producto (Quadros et al., 2001). Además, a nivel social, las empresas innovadoras mejoran los salarios y planes de carrera de los empleados (McAdam y Keogh, 2004). En definitiva, las empresas innovadoras mejoran su funcionamiento al incrementar el nivel de flexibilidad y adaptación a las necesidades de sus stakeholders.

En el ámbito académico, la innovación ha ocupado una posición clave en la literatura empresarial (Herrmann, 2005; Nag, Corley y Gioia, 2007), pues el número de artículos sobre este tópico ha crecido exponencialmente desde la década de 1990, tal como indica Keupp, Palmié y Gassmann (2012). Al mismo tiempo, aumentan las pesquisas sobre los determinantes o antecedentes de la innovación. El gráfico siguiente manifiesta el incesante tratamiento de la literatura sobre los tópicos «estructura organizativa» e «innovación», dos tópicos relevantes que dan sustento a esta investigación.

Así el tratamiento científico, permite la comprensión de la innovación empresarial (Teece, 1996; Teece, 2016) y la demostración de limitados estudios relativos al conocimiento de los tipos de innovación que persiguen las pymes (Oke, Burke y Myers, 2007; Brunswicker y Vanhaverbeke, 2015) y

\footnotetext{
* Nota de agradecimiento: Este artículo versa en un proyecto de investigación de la Confederación de Entidades para la Economía Social de Andalucía (CEPES-Andalucía) financiado por la Dirección General de Economía Social de la Junta de Andalucía (Planes y-ARCO y e-IRIS), implementados con el objetivo de conocer las necesidades sectoriales de innovación y nuevas tecnologías de la información y la comunicación. Los autores agradecen a CEPES-Andalucía y a las empresas participantes el apoyo prestado.
} 
sus antecedentes (Damanpour y Aravind, 2012). A su vez, ello evidencia que las contingencias y los efectos se hallan todavía en menoría (Macher y Mowery, 2009; Keupp et al., 2012); específicamente, De Mello, Marx y Salerno (2012) afirman que la estructura interna de las empresas es un descriptor inexplorado en la literatura sobre innovación.

\section{Gráfico 1. Número de artículos publicados sobre los tópicos "Organizational Structure" e "Innovation"}

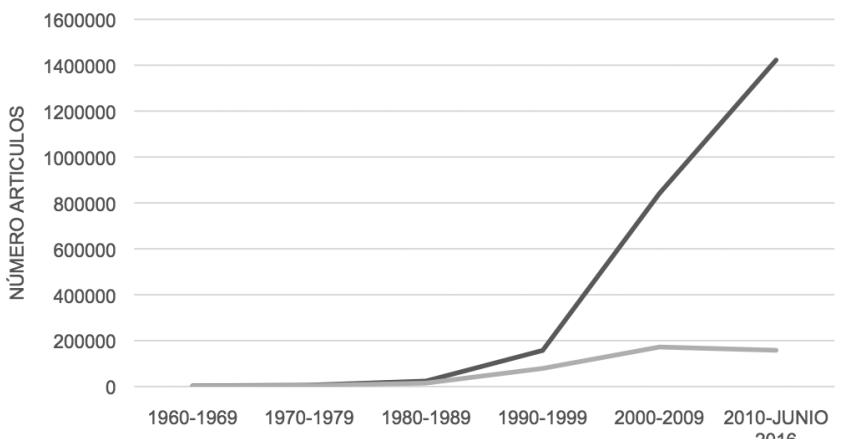

FUENTE: Abi Inform, 2016.

\section{Gráfico 2. Número de artículos publicados con la combinación de los tópicos "Innovation \& Organizational Structure"}

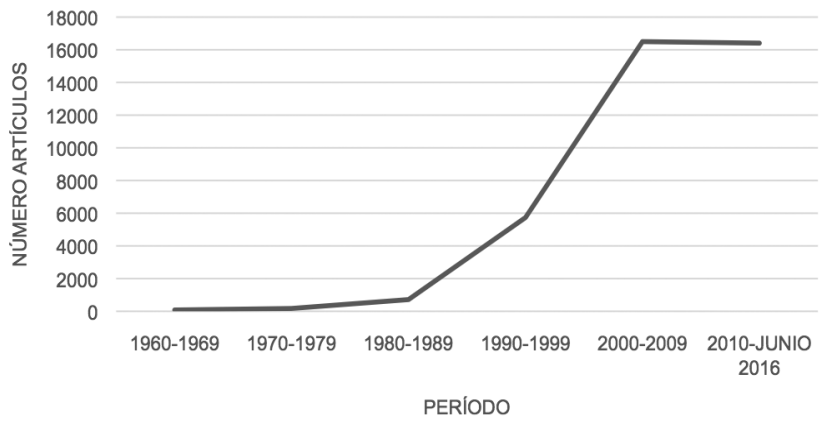

FUENTE: Abi Inform, 2016.

En relación al estado de la literatura sobre el tópico «innovación», ostenta un amplio abanico de debilidades en sus enfoques, los modelos conceptuales considerados, los métodos de captación de datos, los resultados obtenidos e implicaciones teóricas y empíricas. Lícitamente, la principal debilidad de los enfoques es convivir de manera fragmentada (Andries y Debackere, 2006; Fagerberg y 
Verspagen, 2009), tal como apoyan Mosquet, Russo, Wagner, Zablit y Arora (2014) al afirmar la falta de integración de las corrientes de investigación sobre innovación. Con objeto de subsanar este problema, ya partió la Teoría de las Configuraciones de Walker (2008).

Seguidamente, en lo referido a los modelos conceptuales, son escasos los artículos que se centran en la totalidad de tipologías de innovación. Con carácter general, las tipologías de innovación más consideradas en la literatura son producto y proceso (Gunday et al., 2011); siendo, según Piening y Salge (2015), la gestión de innovación de producto la que recibe una mayor atención. En la misma línea, Mosey (2005) y Oke et al. (2007), afirman que no se ha investigado empíricamente las innovaciones radicales e incrementales en las pymes; unido, a que los factores determinantes de la innovación conviven y compiten en conceptualizaciones diversas (Fagerberg y Verspagen, 2009).

Conforme a las debilidades de los métodos de captación de datos, resaltar que los objetos materiales prioritarios se refieren a la innovación en las grandes empresas (Teece, 2016), y existe, en palabras de Oke et al. (2007), una insuficiencia de estudios relacionados con los tipos de innovación en las pymes. Comparten premisa Del Águila y Padilla (2010) cuando aseveran que se ha estudiado, relativamente poco, la innovación en el sector de la «Economía Social». Además, según Mosquet, et al. (2014), la mayoría de los estudios tienden a basarse en muestras pequeñas siendo notables las excepciones.

Los resultados de la investigación sobre innovación presentan un alto grado de inconsistencia y contradicción (Damanpour y Gopalakrishnan, 2001; Keupp et al., 2012). Este carácter discordante y débil, se pone de manifiesto cuando Tidd (2001) afirma que, tras décadas de investigación sobre gestión de la innovación, se han podido entregar resultados estables para un asesoramiento coherente a los gerentes e identificar buenas prácticas. Mientras, se opone Gupta, Tesluk y Taylor (2007), al certificar que, pese a los numerosos estudios sobre el proceso de innovación, los investigadores no han sido capaces de identificar un proceso arquetipo conciso.

Por último, respecto a las implicaciones teóricas de la investigación sobre gestión de la innovación, existen muchas cuestiones aún poco entendidas, puesto que, según Keupp et al. (2012), persisten las lagunas de conocimiento y las fragilidades admitidas. En lo que se refiere a las implicaciones empíricas, Drazin y Schoonhoven (1996) afirman que los ejecutivos se enfrentan a una literatura abrumadoramente teórica y compleja sobre gestión de la innovación; lo que convierte la gestión de la innovación en una tarea desalentadora. Con el objeto de resolver estas inconsistencias, Keupp et al. (2012) atestiguan que las líneas futuras de investigación deberían estar orientadas a generar conocimiento, entre otros temas, sobre la relación causal entre la organización interna y la innovación.

Un panorama similar al referido puede observarse en el sector de Economía Social, campo delimitado conceptualmente y que identifica entre sus actores principales a las cooperativas (Chaves, Monzón y Zaragoza, 2013), referencia de esta investigación. Según Morales y García (2007), el sector de Economía Social muestra indicadores de innovación similares al sector de las pymes, revelando 
que: el éxito de la innovación se restringe a un número reducido de empresas (alrededor del 10\%); los tipos de innovación más utilizados son la innovación de proceso y producto; las fuentes de información más recurridas como orígenes de innovación son la asistencia a ferias/congresos y la propuestas/quejas de clientes. Además, en este marco, resaltar el escaso número de estudios sobre gestión de la innovación en el sector de Economía Social que contrasta con la contribución de este sector al desarrollo sostenible del territorio (Rodríguez y Guzmán, 2013; Gómez y Alexandre, 2014). Así, estas comprobaciones, entre otras, generan el interés específico de indagar sobre la innovación en las empresas de Economías Social, junto, explícitamente, al reconocimiento previo de una literatura débil, y su consecuente búsqueda de mejora.

Se halla interés científico, al mismo tiempo, en la generación de conocimientos a partir de la necesidad corporativa de adquirir y reciclar el know-how e innovar, en palabras de Barzi, Cortelezzi, Marseguerra y Zoia (2015), una necesidad que determina la dominante toma de conciencia de los directivos sobre la innovación fundada por un entorno competitivo de avances tecnológicos y globalización. Reconocida esta pesquisa general, se hallan estudios precisos que señalan que la pertenencia a las cooperativas influyen significativamente en la intensidad del uso de las tecnologías (Birhanu, Girma y Puskur, 2016); el que señala la influencia de la innovación en la actividad exportadora de las sociedades cooperativas (Medina, Mozas, Bernal, y Moral, 2014), así como, la asociación de los beneficios de rendimiento a un mayor enfoque en la gestión de la innovación (Brunswicker y Vanhaverbeke, 2015).

Y finalmente, se descubre utilidad en la actualidad económica cuando una economía mundial como Corea del Sur, compite hoy por un mayor enfoque en empresas sociales de menor tamaño (Kim, 2015). Implicando, a su vez, objetivos socioeconómicos, el alza de la perspectiva del interés social tal como sucede con la parte empresarial y la parte de no mercado del sector de Economía Social (Chaves y Monzón, 2001).

Dentro de la economía real, y la diferenciación entre empresas sociales y sociedades capitalistas, Clemente, Díaz, González, Marcuello y Nachar, (2014) identifican como factor clave de supervivencia empresarial en tiempos de crisis económica a la innovación. Ante esta nueva demonstración de contraste entre tipos de empresas y la distinción entre periodo pre y post crisis, surge el planteamiento base de esta investigación, indagar un comportamiento empresarial determinado en el periodo precrisis.

Un contexto que lleva a señalar, taxativamente, que este trabajo continúa averiguaciones ya reconocidas ${ }^{1}$ y de actual interés científico, es decir, este artículo complementa a dos publicaciones. En particular, con el propósito de subsanar las debilidades observadas en la literatura y facilitar a los direc-

1.- Espasandín, F., Casanueva, C. \& Ganaza, J.D. (2004): "Las nuevas tecnologías de la información y la comunicación en la economía social andaluza", CIRIEC-España, Revista de Economía Pública, Social y Cooperativa, 49, 193-215.

Pérez-Suárez, M., \& Espasandín, F. (2014): "El proceso de internacionalización y los resultados de la innovación en las empresas de economía social andaluza", CIRIEC-España, Revista de Economía Pública, Social y Cooperativa, 82, 65-95. 
tivos de las empresas de Economía Social la gestión de la innovación, los objetivos de este trabajo son dos: conocer la relación entre los parámetros de diseño organizativo (centralización, formalización, preparación y coordinación) y los tipos de innovación; y saber si existen configuraciones estructurales en el sector andaluz de Economía Social, y en tal caso, determinar si diferentes configuraciones estructurales generan diferentes tipos de innovación.

La ausencia de indagaciones previas permite clasificar esta investigación de naturaleza explicativa y exploratoria, y se considera que, por ello será antecesora de futuros esfuerzos científicos. Cierto es que, al igual que la mayoría de estudios sobre innovación en las pymes, enfoca su atención en los factores determinantes de los tipos de innovación.

Asimismo, sigue el enfoque de la Teoría de la Organización, en particular, el Enfoque de Contingencia (Mone, Mckinley y Barker, 1998) y la Teoría de las Configuraciones (Walker, 2008) ampliamente utilizada en la literatura sobre dirección estratégica (Miles, Snow, Meyer y Coleman, 1978; Bensaou y Venkatraman, 1995) para descubrir taxonomías de configuraciones (Lamothe y Dufour, 2007). En último lugar, desde la óptica de los enfoques teóricos de innovación, se integra en las Teorías Estructurales de la Organización, concretamente, en las Teorías Unidimensionales que desarrollan la relación entre las variables estructurales y la innovación, y en las Teorías de Alcance Medio de la Innovación que distinguen entre los tipos (Daft, 1978) y el carácter de innovación (Nord y Tucker, 1987).

La estructura de esta investigación responde a seis apartados: inicialmente, se desarrolla el marco teórico, se plantean y justifican las hipótesis y se diseña el modelo conceptual; luego, se describen los métodos de captación y análisis de datos; se presentan los resultados y se finaliza detallando y discutiendo las conclusiones e implicaciones, acompañadas de limitaciones y futuras líneas.

\section{2.- Marco teórico, planteamiento de las hipótesis y diseño del modelo conceptual}

El contenido de este trabajo se sustenta en dos grandes tópicos: «tipos de innovación empresarial» $y$ «estructura organizativa», entendida la estructura organizativa como factor determinante 0 antecedente de los tipos de innovación. A continuación, se despliega, a partir de la literatura científica consultada, ambos tópicos y se analiza las relaciones que se producen entre ambos. En cuanto a la «innovación», las referencias examinadas ponen de manifiesto que no existe un concepto consensuado en el ámbito científico, tal como evidencia el cuadro siguiente: 


\section{Cuadro 1. Concepto de Innovación}

\begin{tabular}{|l|l|}
\hline Autor & Definición Innovación \\
\hline García y Calantone (2002) & $\begin{array}{l}\text { Comprensión del desarrollo, producción y comercialización de mercado de una invención, } \\
\text { asimismo, la difusión de productos y la adopción por los clientes, }\end{array}$ \\
\hline Luecke y Katz (2003) & $\begin{array}{l}\text { Introducción exitosa de una nueva cosa/método o forma de realización, la combinación o } \\
\text { síntesis de los conocimientos en originales, nuevos productos, procesos o servicios. }\end{array}$ \\
\hline $\begin{array}{l}\text { Nonaka y Toyama (2003); } \\
\text { Tidd (2001) }\end{array}$ & Resultado de la combinación de diferentes conjuntos de conocimiento. \\
\hline $\begin{array}{l}\text { Del Águila y Padilla (2010) } \\
\text { Gunday, Ulusoy, Kilica } \\
\text { y Alpkan (2011) }\end{array}$ & $\begin{array}{l}\text { Medio para cambiar la organización, aplicación comercial de una idea; convertir ideas } \\
\text { en productos, procesos o servicios nuevos o mejorados para dirigirlos al mercado. }\end{array}$ \\
\hline Rodríguez y Guzmán (2013) & $\begin{array}{l}\text { Exordio de un nuevo producto o servicio, las mejoras en aquellos productos que ya existen, } \\
\text { la entrada de un nuevo proceso de producción, la puesta en práctica de un nuevo método } \\
\text { de comercialización u organizacional. }\end{array}$ \\
\hline
\end{tabular}

FUENTE: Elaboración propia.

En base a la enumeración conceptual antepuesta, se considera la innovación como la capacidad organizacional para desarrollar procesos y/o producir y comercializar un producto y/o servicio en el mercado, abordando un procedimiento que parte de una invención y culmina en su adopción por los clientes. Es un intangible empresarial derivado de la aplicación, comprensión, introducción, combinación y transformación.

Según la literatura consultada, el constructo innovación presenta tres características que se detallan a continuación. En primer lugar, presenta un carácter multidimensional (Tidd, 2001). Según Subramanian y Nilakanta (1996), cualquier medida valida de innovación debe considerar varios tipos de innovación, el momento en que se adopta y la consistencia de los patrones que guían su adopción en el tiempo.

En segundo lugar, según De Mello et al. (2012), la innovación es una actividad compleja (Damanpour, 1996) y multidisciplinar (Cuevas-Rodríguez, Cabello Medina y Carmona Lavado, 2014; Story, Daniels, Zolkiewski y Dainty, 2014), pues el conocimiento sobre esta materia se genera por autores que pertenecen a disciplinas tan dispares como ingeniería, economía, sociología, comportamiento organizacional y marketing. Sin embargo, esta investigación interdisciplinaria no se ha sintetizado, siendo por tanto, el desarrollo de una agenda de investigación multidisciplinaria.

En tercer lugar, involucra varias áreas funcionales de la empresa (marketing, producción, finanzas, etc.) así como a sus clientes y proveedores (De Mello et al., 2012). 
Definitivamente, en palabras de Pini y Santangelo (2005), el constructo innovación es una actividad heterogénea puesto que las innovaciones de producto y proceso están condicionadas por diferentes factores.

Con carácter general, los tópicos sobre la innovación han tendido a concentrarse en tres temas básicos: procesos, tipos y antecedentes. De forma más específica, Keupp et al. (2012) indican que la investigación en innovación trata los siguientes subtemas: tipos de innovación (García y Calantone, 2002), innovación abierta (Dahlander y Gann, 2010), relación entre capital social e innovación (Zheng, 2010), medición de los insumos y los resultados de los procesos de innovación (Adams, Bessant y Phelps, 2006), contingencias ambientales (Tidd, 2001), relación entre innovación y productividad nacional (Denyer y Neely, 2004), desarrollo de nuevos productos (Page y Schirr, 2008), los aspectos cognitivos individuales de la innovación (Anderson, De Dreu y Nijstad, 2004), rol de terceros en el proceso de innovación (Bogers y West, 2010), difusión de la innovación (O’Neill, Pouder y Buchholtz, 1998), creación de redes (Pittaway, Robertson, Munir, Denyer y Neely, 2004), relación entre la orientación al mercado y la innovación (De Luca, Verona y Vicari, 2010) y el papel del tamaño en la organización (Camisón y Boronat, 2004).

Particularizando, esta investigación, que forma parte del subtema tipos de innovación, distingue diferentes criterios para clasificar la innovación. Tradicionalmente, Boer y During (2001) clasifican la innovación atendiendo a varios criterios: el objeto de la innovación (innovaciones producto y de proceso); su impacto (innovación incremental y radical); el efecto de la innovación; la escala en la que se realiza; su origen y la naturaleza. De forma complementaria el Manual de Oslo de la OCDE (2005), identifica cuatro tipos diferentes de innovación: innovación de producto, innovación de proceso, innovación de marketing e innovación organizacional. En esta línea, se consideran las innovaciones de producto, proceso, incremental y radical; hecha la acotación, se define brevemente cada una de ellas.

El Manual de Oslo define la innovación de producto como la introducción de un buen servicio nuevo o significativamente mejorado con respecto a sus características o usos previstos, incluyendo mejoras significativas en especificaciones técnicas, componentes y materiales, software incorporado, la facilidad de uso u otras características funcionales (OCDE, 2005).

Oke et al. (2007) consideran que la innovación de proceso implica la creación y/o mejora de los medios de producción, servicios o las operaciones administrativas, así como, la evolución de los procesos, sistemas y actividades de reingeniería comprometidos para el desarrollo de nuevos productos. Tras estas definiciones, cabe señalar, desde la óptica del objeto de la innovación, que Myers y Marquis (1969) afirman que las empresas, por término medio, adoptan más innovaciones de producto que de proceso. Sin embargo, García y Molero (2010), afirman que el tipo de innovación que se hace en España tiene mayor orientación hacia los procesos.

Por otra parte, INE (2000) define la innovación incremental como la mejora de productos o procesos ya existentes que aportan menor novedad; y la innovación radical es aquella que supone la intro- 
ducción en el mercado de un producto y/o proceso totalmente nuevo ya que presenta diferencia significativas a los productos existentes en cuanto a finalidad, características y componentes (Manzini, 2014). Damanpour (1996) puntualiza que las innovaciones radicales se adoptan, por término medio, con menos frecuencia que las innovaciones incrementales. Comparten afirmación Oke et al. (2007), cuando identifican que las pymes de Reino Unido están enfocadas hacia la innovación incremental.

Nuevamente, en general, la investigación sobre los tipos de innovación no suele orientarse a todos los tipos de innovación referidos. Entre las referencias consultadas, únicamente el trabajo de Hoonsopon y Ruenrom (2012) distingue entre innovación de producto radical e innovación de producto incremental. Entendiendo por innovación de producto radical el desarrollo de producto que tiene un conjunto diferente de características y atributos de rendimiento que crean un conjunto de beneficios diferentes al de los productos existentes desde la perspectiva del cliente. Y por innovación de producto incremental, el desarrollo de productos que tienen pequeñas cambios en los atributos y los beneficios son mínimos desde la perspectiva del cliente.

Lógicamente, conseguir resultados de la innovación reclama un proceso de alineación de la innovación que no es sencillo de gestionar, puesto que, hay innumerables maneras de organizar. En este sentido, Andrew y Sirkin (2008) consideran que las empresas innovadoras siguen seis practicas: en primer lugar, designar a una sola persona con responsabilidad operativa para conducir o facilitar innovación; en segundo lugar, generar pequeños unidades que trasladen las ideas al ámbito comercial; en tercer lugar, crear y compartir una cultura y un vocabulario de la innovación; en cuarto lugar, implantar las condiciones necesarias para la innovación (tiempo, espacio, motivación, etc.); en quinto lugar, estar abierto a las ideas del entorno; y en sexto lugar, realizar la evaluación y el seguimiento de medidas significativas sobre los resultados de la innovación. Sin embargo, los resultados de la innovación no solo están condicionados por una buena organización, son numerosos los factores determinantes 0 antecedentes de la innovación.

En este sentido, si bien se ha avanzado en la identificación de determinantes, aún hay mucha confusión debido a los problemas de definición (West y Farr, 1989) y los resultados de estudios empíricos (Damanpour, 1991). Estos determinantes se ubican en el plano individual (Mcmurray y Williams, 2004) y organizacional (Rodríguez y Guzmán, 2013; entre otros).

En el ámbito organizacional, la literatura consultada identifica determinantes relacionados con el pensamiento innovador (Shapiro, 2001), el comportamiento innovador (Baard y Watts, 2007) y los tipos de innovación (Walker, 2008). Recientemente, Rodríguez y Guzmán (2013) afirmaban que la innovación depende de factores externos e internos. Los factores externos proceden del entorno de la empresa, y entre ellos, se considera: el sector y el tipo de industria (Hao, Kasper y Muehlbacher, 2012), el carácter nacional o extranjero de la propiedad (Love y Ashcroft, 1999), el acceso a la financiación externa (Del Águila y Padilla, 2010) o la pertenencia a un grupo de empresas (Aschhoff y Schmidt, 2008). Los factores internos se refieren a las características de la empresa y del empresario, y entre ellos, se mencionan los siguientes: orientación al mercado (Liu, 1995), acomodos empresariales 
(Atuahene-Gima y Ko, 2001), comportamiento estratégico (Wang, 2008), tácticas (Gatignon y Xuereb, 1997), presencia de I+D (Love y Ashcroft, 1999), grado de internacionalización (González-Pernía y Peña-Legazkue, 2007), capacidades de la organización (Hoonsopon y Ruenrom, 2012), cultura organizativa (García-Del Junco, Espasandín-Bustelo, Palacios-Florencio y Dutschke-Jeremias, 2013), y características estructurales como el tamaño (Camisón y Boronat, 2004), edad (Sorensen y Stuart, 2000), la estructura de la propiedad (Acha, Gann y Salter, 2005) y la estructura organizativa (Hao et al., 2012).

Por otra parte, en el sector de la Economía Social, Del Águila y Padilla (2010) estudian diversos determinantes de la innovación. En particular, consideran determinantes internos (antigüedad de la empresa, tamaño, actitud estratégica, apoyo de la dirección, nivel educativo, barreras de la innovación y estrategia de la innovación) y externos (sector de actividad). Compartido es ello por Rodríguez y Guzmán (2013) al analizar los determinantes de la innovación en las cooperativas españolas, pues toman como determinantes internos, el capital social, el tamaño y la estrategia de negocio; y como determinantes externos, el sector y la localización del territorio. Se concluye, por tanto, que no se conocen investigaciones que estudien la estructura organizativa como un factor determinante de los tipos de innovación en el ámbito de la Economía Social.

Una vez planteada la revisión general de la literatura relacionada con los dos tópicos, «estructura organizativa» y «tipos de innovación», y tomando en consideración la afirmación de Walker (2008) sobre que "las relaciones entre los antecedentes y los tipos de innovación son relativamente complejas" junto a las evidencias adyacentes, se procede a justificar y plantear las hipótesis y el modelo conceptual.

En palabras de DeCanio, Dibble y Amir-Atefi (2000), son diversas las disciplinas desde las que se enfoca el estudio de la estructura organizativa. Aunque esta multidisciplinariedad podría implicar importantes diferencias conceptuales, la mayoría de los investigadores (Nahm, Vonderembse y Koufteros, 2003; Robbins y Coulter, 2005; Mintzberg, 2012) se aproximan a la definición que propone Daft (2012), según la cual la estructura organizacional es un marco de referencia que identifica la forma en que se ejecutan las tareas, se distribuyen los recursos, y se consigue la coordinación entre los diferentes departamentos y divisiones para lograr objetivos específicos. De igual modo, para Hao et al. (2012), la estructura organizativa es un constructo multidimensional que se refiere a la división del trabajo (especialización, diferenciación, centralización, complejidad) y los mecanismos de coordinación (la normalización, la formalización y la flexibilidad). También, Mintzberg (1996) refleja este carácter multidisciplinar en nueve parámetros de diseño referidos al esquema de puestos de trabajo (la especialización, la formalización del comportamiento, la preparación y el adoctrinamiento), al diseño de la superestructura (agrupación de unidades y tamaño de la unidad), al diseño de vínculos laterales (sistemas de planificación y control, y dispositivos de enlace) y diseño del sistema decisor (centralización). Lo cierto, es que se exalta la afirmación de Matheson (2009) sobre que la formalización del comportamiento y la centralización son los parámetros de diseño clave de la estructura. 
Puntualizar aún más, Cosh, Fu y Hughes (2012) y Palmer y Wright (2010), entre otros, consideran que la centralización se refiere al grado en el que el poder de decisión se concentra en la parte superior de la jerarquía organizativa y la formalización del comportamiento al grado en que los puestos de trabajo están estandarizados en las organizaciones. Igualmente, se consideran las premisas propuestas por García-Del Junco et al. (2013), quienes definen la centralización como el grado en que todos los empleados participan en la toma de decisiones y la formalización del comportamiento como el grado en que las políticas, procedimientos y reglas de la empresa se establecen por escrito.

Centrando estos dos conceptos, la literatura consultada pone en evidencia que la formalización del comportamiento se relaciona negativamente con los tipos de innovación. En este sentido, Damanpour (1991) y Brandyberry (2003) afirman que la formalización del comportamiento es un determinante negativo de la innovación organizacional y, Cohn y Turyn (1980) encuentran que también existe una relación negativa entre la formalización del comportamiento y la adopción de innovación de procesos.

En consecuencia, esto nos permite formular la siguiente hipótesis:

$H_{1}$. La formalización del comportamiento es menor en las empresas de Economía Social que innovan.

Según Jansen, Van den Bosch y Volberda (2006), la centralización se ha estudiado ampliamente en la literatura sobre innovación y la relación entre la toma de decisiones y los diferentes tipos de innovación aunque siguen presentando escasa claridad (Cardinal, 2001). Sin embargo, son numerosos los autores que afirman que la centralización influye negativamente en los tipos de innovación, así apoyan esta hipótesis Brandyberry (2003) y Hassan, Shaukat, Nawaz y Naz (2013), quienes aseveran que la centralización disuade la innovación; mientras que Damanpour (1991) sostiene que la centralización influye negativamente en la capacidad de innovación, específicamente, en la innovación exploratoria (Jansen et al., 2006). A la luz de estas evidencias, formulamos la siguiente hipótesis:

$\mathrm{H}_{2}$. La centralización de la estructura organizativa es mayor en las empresas de Economía Social que no innovan.

Además, el carácter multidimensional de la estructura reflejado en los parámetros de diseño (Mintzberg, 1996) origina distintos arquetipos estructurales. En este ámbito, resaltan los trabajos de Burns y Stalker (1961), quienes distinguen estructuras mecanicistas y orgánicas; Mintzberg (1996) identifica cinco configuraciones estructurales (estructura simple, burocracia maquinal, burocracia profesional, forma divisional y adhocracia), y más recientemente, Matheson (2009) propone ocho configuraciones estructurales (profesional, misionera, maquinal, diversificada, en red, política, autocrática y carismática). Sobre ello y señalando a Burns y Stalker (1961), las estructuras orgánicas son menos formalizadas, más flexibles, más creativas y adaptadas a la innovación. Esta evidencia ampliamente citada en la literatura permite formular la siguiente hipótesis: 
$H_{3}$. La estructura orgánica es más característica de las empresas de Economía Social que innovan.

Mintzberg (1996) afirma que la estructura simple y la adhocracia son las configuraciones con mayor capacidad de innovación y adaptación, mientras que las otras tres configuraciones estructurales (burocracia maquinal, burocracia profesional y forma divisional) tienen capacidades innovadoras más limitadas, $y$ responden peor a la novedad y al cambio. A la luz de este argumento, formulamos la siguiente hipótesis:

$\mathrm{H}_{4}$. La estructura organizativa de las empresas de Economía Social que no innovan difiere de la estructura organizativa de las empresas de Economía Social que innovan.

Desde otro punto de vista, Culebras (2010), basándose en los datos de la Encuesta sobre Innovación Tecnológica Empresarial en 2006 y en las características tecnológicas y estructurales de las organizaciones, identifica ocho patrones de innovación en las empresas españolas: Patrón Estrella (el más innovador, de mayor apertura externa, constituido principalmente por grandes empresas), Patrón Brillante (de mayor radicalidad innovadora, elevada apertura externa, constituido principalmente por pymes), Patrón de Procesos (de mayor innovación de proceso), Patrón de Nuevos Productos (de mayor creación de empresas, orientado a la innovación de producto), Patrón Medianamente Innovador con apropiación de la innovación, Patrón Medianamente Innovador, Patrón Poco Innovador (poco innovador, seguido principalmente por medias y grandes empresas) y Patrón de Menor Compromiso Innovador. Entendiendo que una situación similar se observa en el caso de las empresas de Economía Social innovadoras, se formula la siguiente hipótesis:

$H_{5}$. Las características o parámetros de diseño de la estructura organizativa revelan la existencia de diferentes tipos o grupos estratégicos en el colectivo de empresas de Economía Social que innovan.

Como ya se menciona previamente, la literatura sobre innovación no suele enfocarse en detalle en la estructura organizativa como un determinante de la innovación y suele centrar su atención, a lo sumo, en un criterio y/o en un tipo o resultado de innovación. No se conocen, por tanto, investigaciones que relacionen los tipos de estructura organizativa con los tipos de innovación, e identificando como importante esta relación se enuncia la siguiente hipótesis.

$H_{6}$. Los grupos estratégicos identificados en el colectivo de empresas de Economía Social que innovan generan diferentes tipos de innovación.

A modo de síntesis, a continuación se presenta el modelo conceptual de la investigación que sintetiza gráficamente las hipótesis planteadas y justificadas con antelación. 


\section{Gráfico 3. El Modelo Conceptual}

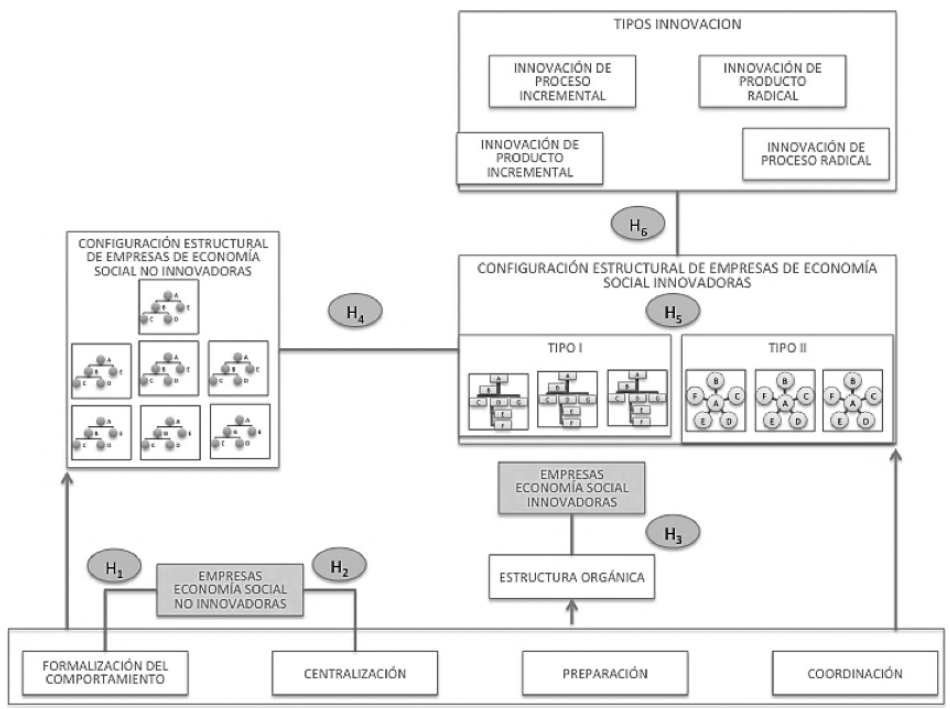

FUENTE: Elaboración propia.

\section{3.- Metodología}

Definido el objetivo, se realizó una clasificación de las empresas en grupos para, posteriormente, estudiar detenidamente esos perfiles teniendo en cuenta la estructura organizativa y los tipos de innovación las empresas andaluzas de Economía Social. De esta manera, este apartado versa sobre el método de captación y tratamiento de datos y, en particular, contempla detallados los parámetros de diseño, la descripción de la población y la muestra así como los métodos estadísticos empleados.

Seguidamente, se acudió al tratamiento de una fuente primaria acopiando una serie de datos seleccionados del tejido empresarial protagonista con el objeto de abordar una retrospección. En consonancia, para medir las variables seleccionadas, se diseñó un cuestionario semiestructurado de 10 preguntas, testado por los grupos de investigación de la Universidad de Sevilla: SEJ301: PYMES, gestión y competitividad y SEJ230: Planificación y Análisis Económico. Para posteriormente, recopilar los datos necesarios de una muestra representativa del sector de Andalucía como estipula la ficha técnica. 


\subsection{Descripción de la población y muestra}

Este análisis comenzó delimitando un objeto material: las empresas-cooperativas y sociedades laborales-que integran el sistema andaluz de Economía Social, delimitación poblacional en analogía a otras investigaciones (Clemente, Díaz, González, Marcuello y Nachar, 2014). En este sentido, el Ministerio de Trabajo e Inmigración, cifró en 5543 el número total de sociedades activas-cooperativas y sociedades laborales-en 2002 (Clemente, Díaz y Marcuello, 2008). En la siguiente descripción técnica, se incluyen los datos compilados del estudio cuya recogida se realizó en 2003.

\section{Tabla 1. Ficha Técnica}

\begin{tabular}{|l|l|}
\hline Metodología & 2002 \\
Proceso metodológico & Entrevista Personal. \\
Tipo de preguntas & Dicotómicas, Nominales e Intervalos. \\
Universo poblacional & 5543 empresas andaluzas de Economía Social. \\
Muestra obtenida & 515 empresas andaluzas de Economía Social. \\
Forma de contacto & Correo electrónico y teléfono. Encuentro Presencial. \\
Método de muestreo & Muestreo aleatorio simple para proporciones con un nivel de confianza del \\
& $95 \%$ y con el supuesto de máxima indeterminación $(p=q=0,5)$. \\
Error & $\pm 4,11 \%$ \\
Fecha de encuestación & Enero a Junio de 2003. \\
\hline
\end{tabular}

FUENTE: Elaboración propia.

\subsection{Selección de los métodos estadísticos}

La recogida de datos se realizó a través de un cuestionario estructurado de naturaleza personal durante 2003. Datos tratados en SPSS/PC (V23) generando la información necesaria. Asimismo, la extracción del elemento muestral (515) se realiza mediante un procedimiento de muestreo aleatorio simple, fundamentado en el azar y aplicado mediante tablas de números aleatorios. Por último, señalar que se procedió a eliminar del análisis las respuestas desconocidas.

Para contrastar las hipótesis que se formulan, se eligió el método estadístico descriptivo e inferencias- frecuencias, tablas de contingencia, contrastes de hipótesis en base a la prueba z y la prueba t para contrastar medias- en la determinación de cuatro variables tipo escala de Likert del 1 al 7 ; y el análisis clúster (Dopico, Rodríguez y González, 2012; Chaudhry, 2013; Pérez y Valiente, 2015). El análisis clúster siguió un procedimiento de pasos heterogéneos (Díaz, 1998): estudio pormenorizado de las variables a incluir; uso de un método de clasificación de clúster con objeto de crear grupos similares; cálculo de las distancias o similitudes, y validación de los resultados. Próximamente, se muestran las variables estudiadas, su concepto y medición. 


\section{Tabla 2. Definición de las Variables observadas}

\begin{tabular}{|c|c|c|c|}
\hline Denominación & \multicolumn{2}{|c|}{ Concepto que expresa } & Medida \\
\hline Formalizar & \multicolumn{2}{|c|}{ Grado de formalización de los puestos de trabajo } & 1 a 7 \\
\hline PrepMaxRes. & \multicolumn{2}{|c|}{ Grado de preparación del máximo responsable } & 1 a 7 \\
\hline PrepEquipo. & \multicolumn{2}{|c|}{ Grado de preparación del equipo } & 1 a 7 \\
\hline PrepTrabaj. & \multicolumn{2}{|c|}{ Grado de preparación del trabajador } & 1 a 7 \\
\hline Coordinación & \multicolumn{2}{|c|}{ Grado de funcionamiento coordinado de las unidades } & 1 a 7 \\
\hline Centralización $\quad G$ & \multicolumn{2}{|c|}{ Grado de descentralización } & 1 a 7 \\
\hline $\begin{array}{l}\text { Empresas con innovación } \\
\text { incremental de proceso }\end{array}$ & \multicolumn{2}{|c|}{$\begin{array}{l}\text { Implica la creación y/o mejora gradual de los medios } \\
\text { de producción, servicios o las operaciones administrativas } \\
\text { comprometidos para el desarrollo de nuevos productos. }\end{array}$} & Dicotómica (Sí/No) \\
\hline $\begin{array}{l}\text { Empresas con innovación } \\
\text { radical de proceso }\end{array}$ & \multicolumn{2}{|c|}{$\begin{array}{l}\text { Implica la creación y/o mejora sustancial de los medios } \\
\text { de producción, servicios o las operaciones administrativas } \\
\text { comprometidos para el desarrollo de nuevos productos. }\end{array}$} & Dicotómica (Sí/No) \\
\hline $\begin{array}{l}\text { Empresas con innovación } \\
\text { radical de producto }\end{array}$ & \multicolumn{2}{|c|}{$\begin{array}{l}\text { Desarrollo de producto que tiene un conjunto diferente } \\
\text { de características y atributos de rendimiento que crean } \\
\text { un conjunto de beneficios diferentes al de los productos } \\
\text { existentes desde la perspectiva del cliente. }\end{array}$} & Dicotómica (Sí/No) \\
\hline $\begin{array}{l}\text { Empresas con innovación } \\
\text { incremental de producto }\end{array}$ & \multicolumn{2}{|c|}{$\begin{array}{l}\text { Desarrollo de productos que tienen pequeños cambios } \\
\text { en los atributos y los beneficios son mínimos desde la } \\
\text { perspectiva del cliente. }\end{array}$} & Dicotómica (Sí/No) \\
\hline \multirow[b]{2}{*}{ Forma Jurídica } & \multirow[b]{2}{*}{$\begin{array}{l}\text { Clasificación de las empresas } \\
\text { en función de la legislación } \\
\text { aplicable a la misma }\end{array}$} & \multicolumn{2}{|l|}{ Medida Nominal } \\
\hline & & \multicolumn{2}{|c|}{$\begin{array}{l}\text { Cooperativas de cooperación } \\
\text { Cooperativas de explotación comunitaria } \\
\text { Cooperativas de integración } \\
\text { Cooperativas de seguros } \\
\text { Cooperativas de trabajo asociado } \\
\text { Cooperativas de vivienda } \\
\text { Cooperativas de consumidores } \\
\text { Cooperativas de segundo o ulterior grado } \\
\text { Cooperativas de servicios } \\
\text { Cooperativas de interés social } \\
\text { Cooperativas de educación } \\
\text { Sociedad Limitada Laboral }\end{array}$} \\
\hline \multirow[b]{2}{*}{$\begin{array}{l}\text { Ciclo de vida de } \\
\text { la Empresa (Edad) }\end{array}$} & \multirow[b]{2}{*}{$\begin{array}{l}\text { Número de años que } \\
\text { lleva la empresa activa }\end{array}$} & Medida de Intervalo & \\
\hline & & $\begin{array}{l}\text { 0-1 años } \\
2-3 \text { años } \\
4-7 \text { años } \\
\text { 8-11 años } \\
12-15 \text { años } \\
16-19 \text { años } \\
\text { Más de } 20 \text { años }\end{array}$ & \\
\hline
\end{tabular}




\begin{tabular}{|c|c|c|}
\hline \multirow[t]{2}{*}{ Sector Económico } & \multirow{2}{*}{$\begin{array}{l}\text { División de las actividades } \\
\text { económicas en función } \\
\text { de áreas homogéneas }\end{array}$} & Medida Nominal \\
\hline & & $\begin{array}{l}\text { Sector primario } \\
\text { Sector secundario } \\
\text { Sector terciario } \\
\end{array}$ \\
\hline \multirow[b]{2}{*}{ Tamaño de la Plantilla } & \multirow[b]{2}{*}{ Número de empleados } & Medida de Intervalo \\
\hline & & $\begin{array}{l}\text { Menos de } 9 \\
10-49 \\
50-249 \\
250-499 \\
\text { Más de } 499 \\
\end{array}$ \\
\hline \multirow[b]{2}{*}{ Tamaño de Socios } & \multirow[b]{2}{*}{ Número de socios } & Medida de Intervalo \\
\hline & & $\begin{array}{l}\text { Menos de } 9 \\
10-49 \\
50-249 \\
250-499 \\
\text { Más de } 499 \\
\end{array}$ \\
\hline \multirow[b]{2}{*}{$\begin{array}{l}\text { Volumen de } \\
\text { Facturación }\end{array}$} & \multirow[b]{2}{*}{ Total de Ventas anuales $(€)$} & Medida de Intervalo \\
\hline & & $\begin{array}{l}\text { Menos } 60.101,21 \\
60.101,21 \text { a } 150.253,03 \\
150.253,03 \text { a } 300.506,05 \\
303.516,17 \text { a } 601.012,10 \\
607.022,23 \text { a1.202.24,21 } \\
1.208 .034,33 \text { a } 3.005 .060,52 \\
3.011 .070,64 \text { a } 6.010 .121,04 \\
\text { Más de } 6.010 .121,04\end{array}$ \\
\hline
\end{tabular}

FUENTE: Elaboración propia.

En primer lugar se dividió la población en dos, empresas que sí innovan y empresas que no innovan, resultando una variable dicotómica (Si/No: innovación empresarial) (López, Montes y Vázquez, 2003; Díaz, Aguiar y De Saá, 2006). Después de esta división, se realizó el análisis clúster dentro de las empresas que innovan y han contestado a todas las preguntas (Martínez, 1984), siendo las preguntas utilizadas para citado análisis las vinculantes a las variables formalización, preparación máximo responsable y centralización (Escala de Likert del 1 al 7). Consecutivamente, se optó por la técnica no jerárquica, pues las variables parten de la matriz original de puntuaciones, se decidió el número de clústers a obtener antes de comenzar el proceso de cálculo y los clústers resultantes son independientes. Es decir, se trató de formar grupos bajo la premisa de maximizar la varianza inter-grupos y minimizar la varianza intra-grupos. Despejado ello, se aplicó el método k-medidas y se calculó la distancia euclidea simple. Dando lugar a un procedimiento que validó diferencias significativas entre dos clústers. 


\section{4.- Resultados}

Inicialmente, describir los datos generales de la muestra considerada. El modelo arquetipo que representa a la muestra (ver Tabla 3), permite concluir que el sistema de empresas de Economía Social analizado, está integrado por pequeñas cooperativas y sociedades laborales que han superado los tres años de actividad; y desarrollan su actividad productiva en el sector primario y terciario.

\section{Tabla 3. Modelo arquetipo de la muestra de empresas de Economía Social}

\begin{tabular}{|l|l|}
\hline Forma Jurídica & Cooperativas y Sociedades Laborales \\
Ciclo de vida de la Empresa (Edad) & $4-7$ ñ̃os \\
Sector Económico & Sector primario y terciario \\
Tamaño de la Plantilla & Menos de 9 trabajadores \\
Tamaño de Socios & Menos de 10 socios \\
Volumen de Facturación $(\boldsymbol{\xi})$ & $60.101,21-150.253,03$ \\
\hline
\end{tabular}

FUENTE: Elaboración propia.

A continuación se muestran los resultados relativos a las hipótesis planteadas en la investigación. En este sentido, la tabla siguiente manifestó la diferencia en los parámetros de diseño (formalización, preparación, coordinación y centralización) y en la estructura organizativa de las empresas de Economía Social que no innovan versus las que innovan; considerándose estas últimas como aquellas que, en los últimos 3 años, han conseguido innovaciones incrementales o radicales de producto o de proceso.

Tabla 4. Empresas que innovan vs Empresas que no innovan: parámetros de diseño de la estructura organizativa

\begin{tabular}{|l|cccc|cccc|}
\hline & \multicolumn{4}{|c|}{ No innova } & \multicolumn{4}{c|}{ Sí innova } \\
\cline { 2 - 9 } & Media & $\begin{array}{c}\text { Mediana } \\
\text { típica }\end{array}$ & Desviación & Moda & Media & Mediana & $\begin{array}{c}\text { Desviación } \\
\text { típica }\end{array}$ & Moda \\
\hline Formalizar & 4,11 & 4,00 & 2,12 & 6,00 & 3,77 & 4,00 & 2,04 & 1,00 \\
PrepMaxRes & 2,91 & 2,00 & 1,51 & 2,00 & 3,41 & 3,00 & 1,76 & 2,00 \\
PrepEquipo & 2,86 & 2,00 & 1,45 & 2,00 & 3,46 & 3,00 & 1,78 & 2,00 \\
PrepTrabaj. & 2,66 & 2,00 & 1,29 & 2,00 & 3,08 & 2,00 & 1,48 & 2,00 \\
Coordinación & 5,46 & 6,00 & 1,53 & 7,00 & 5,77 & 6,00 & 1,46 & 7,00 \\
Centralización & 4,38 & 5,00 & 2,05 & 7,00 & 4,24 & 4,00 & 2,02 & 7,00 \\
\hline
\end{tabular}

${ }^{*}$ p-level<0,05. FUENTE: Elaboración propia. 
A la luz de las evidencias de la tabla previa, fue posible concluir que se sostienen la hipótesis $H_{1}, H_{3} y H_{4}$, pues la formalización del comportamiento es ligeramente menor en las empresas de Economía Social que innovan y, en consecuencia, la estructura es más orgánica. Además, los niveles de preparación de los máximos responsables, de los equipos de trabajo y de los trabajadores son significativamente menores en las empresas de Economía Social no innovadoras y el nivel de coordinación es significativamente inferior en las empresas de Economía Social no innovadoras.

Por otra parte, aunque el nivel de descentralización de las empresas de Economía Social permitió abundar en las diferencias estructurales entre las empresas de Economía Social innovadoras y no innovadoras $\left(H_{4}\right)$, no posibilita confirmar la hipótesis $H_{2}$, pues los niveles de descentralización, contrariamente a lo que se establece en la hipótesis, son mayores en las empresas de Economía Social que no innovan.

Una vez comprobadas las diferencias estructurales entre las empresas de Economía Social innovadoras y no innovadoras, se estudió si los valores de los parámetros de diseño de la estructura organiza permiten identificar grupos estratégicos integrados por empresas de Economía Social con estructuras organizativas similares $\left(H_{5}\right)$.

En este sentido, aclarar que, motivado por el limitado número de empresas que innovan y han respondido a todas las cuestiones de análisis (166), y porque la literatura consultada y referida tiende a polarizar en los extremos los valores de los parámetros de diseño (alto o bajo) y los tipos de estructura (orgánica o mecanicista), se planteó crear 2 grupos estratégicos: el clúster 1, integrado por 90 empresas innovadoras de Economía Social y el clúster 2, integrado por 76 empresas innovadoras de Economía Social (ver Tabla 5).

\section{Tabla 5. Número de empresas en cada clúster}

\begin{tabular}{|l|ccc|}
\hline \multirow{2}{*}{ Clúster } & 1 & 90 & $54,22 \%$ \\
& 2 & 76 & $45,78 \%$ \\
\hline Válidos & & 166 & $100 \%$ \\
\hline
\end{tabular}

FUENTE: Elaboración propia.

\section{Tabla 6. Distancias entre centros de clústeres finales}

\begin{tabular}{|l|c|c|}
\hline Clúster & 1 & 2 \\
\hline 1 & - & 3,763 \\
\hline 2 & 3,763 & - \\
\hline
\end{tabular}

FUENTE: Elaboración propia. 
Con el objeto de validar y confirmar los resultados del análisis clúster, se aplicó un análisis de la varianza (ver Tabla 6), que permitió encontrar diferencias significativas entre cada uno de los clúster, y un análisis discriminante (ver Tabla 7), con el que se conoce que los resultados coinciden en casi todos los casos. Consecuentemente, los dos clúster identificados conforman dos grupos estratégicos de empresas innovadoras de Economía Social con estructuras organizativas diferentes.

\section{Tabla 7. Análisis de la varianza}

\begin{tabular}{|l|cc|}
\hline & $F$ & Sig. \\
\hline Grado de formalización de los puestos de trabajo & 25,931 & 0,000 \\
Grado de preparación del máximo responsable & 24,995 & 0,000 \\
Grado de centralización & 282,250 & 0,000 \\
\hline
\end{tabular}

FUENTE: Elaboración propia.

\section{Tabla 8. Análisis discriminante}

\begin{tabular}{|l|c|cc|}
\hline \multicolumn{2}{|c|}{} & Grupo pronosticado (análisis discriminante) \\
\hline Número de clúster & 1 & 1 & 2 \\
& 2 & 88 & 2 \\
& 2 & 2 & 74 \\
\hline
\end{tabular}

FUENTE: Elaboración propia.

Una vez conocida la existencia de dos grupos estratégicos, se describió, en base a los datos de la tabla siguiente, las características de la estructura organizativa de cada clúster y se designó los clúster. Es preciso comentar que el clúster 1 presentó una media significativamente superior al clúster 2 en todas las variables analizadas a continuación. Concretamente, el clúster 1 se caracteriza por un nivel medio-alto de formalización del comportamiento, un nivel de preparación de los máximos responsables también medio-alto, un nivel medio de preparación de los equipos de trabajo y de los trabajadores moderado y un grado de coordinación y de centralización altos. Todas estas características, excepto el alto grado de centralización, aproximan las empresas que integran este grupo estratégico al modelo de estructura mecanicista.

Por el contrario, las empresas del clúster 2 presentaron niveles medio-altos de coordinación y niveles medio-bajos de formalización del comportamiento, preparación de los máximos responsables, preparación de los equipos de trabajo, preparación de los trabajadores y centralización. En oposición al modelo anterior, se entendió que este grupo estratégico dispone de un modelo próximo a una estructura orgánica, por sus bajos niveles de formalización del comportamiento y a pesar de su bajo grado de centralización. 
En consecuencia, se sostiene la hipótesis $H_{5}$, pues se identificaron con total claridad dos grupos estratégicos en el colectivo de empresas innovadoras de la Economía Social, cuyas estructuras son neta y claramente diferentes.

\section{Tabla 9. Grupos Estratégicos en las empresas de Economía Social que innovan según los parámetros de diseño de la estructura}

\begin{tabular}{|l|cccc|cccc|}
\hline & \multicolumn{3}{|c|}{ Estructura próxima al tipo mecanicista } & \multicolumn{4}{c|}{ Estructura próxima al tipo orgánico } \\
\cline { 2 - 9 } & Media & Mediana & $\begin{array}{c}\text { Desviación } \\
\text { típica }\end{array}$ & Moda & Media & Mediana & $\begin{array}{c}\text { Desviación } \\
\text { típica }\end{array}$ & Moda \\
\hline Formalizar & 4,51 & 5,00 & 2,07 & 7,00 & 2,99 & 3,00 & 1,73 & 1,00 \\
PrepMaxRes & 4,02 & 3,00 & 1,87 & 2,00 & 2,74 & 2,00 & 1,35 & 2,00 \\
PrepEquipo & 3,86 & 3,00 & 1,83 & 2,00 & 3,03 & 2,00 & 1,62 & 2,00 \\
PrepTraba & 3,38 & 3,00 & 1,60 & 2,00 & 2,77 & 2,00 & 1,29 & 2,00 \\
Coordinación & 5,98 & 7,00 & 1,46 & 7,00 & 5,55 & 6,00 & 1,50 & 6,00 \\
Centralización & 5,74 & 6,00 & 1,18 & 7,00 & 2,55 & 3,00 & 1,27 & 3,00 \\
\hline
\end{tabular}

*p-level<0,05. FUENTE: Elaboración propia.

Finalmente, se comprobó si los dos grupos estratégicos generan resultados o tipos de innovación semejantes o diferentes. Al respecto, resaltar, en primer lugar, que las empresas de Economía Social innovadoras de los dos grupos estratégicos identificados se asemejan, pues consiguen un alto número de innovaciones incrementales de proceso y, en segundo lugar, se diferencian puesto que, proporcionalmente, las empresas cuya estructura se aproxima al modelo mecanicista consiguen más innovaciones radicales de producto, y las empresas que se aproximan al modelo de estructura orgánica consiguen significativamente más innovaciones radicales de proceso. 


\section{Tabla 10. Grupos estratégicos y resultados de la innovación}

\begin{tabular}{|c|c|c|c|c|c|}
\hline \multirow{3}{*}{\begin{tabular}{|l} 
\\
$\begin{array}{l}\text { Empresas con innovación } \\
\text { incremental de proceso }\end{array}$
\end{tabular}} & \multirow{3}{*}{$\begin{array}{l}\text { No consiguen } \\
\text { Sí consiguen }\end{array}$} & \multicolumn{2}{|c|}{$\begin{array}{l}\text { Clúster } 10 \text { Empresas de } \\
\text { Economía Social con una estruc- } \\
\text { tura aproximada al tipo mecanicista }\end{array}$} & \multicolumn{2}{|c|}{$\begin{array}{l}\text { Clúster } 2 \text { o Empresas de } \\
\text { Economía Social con una estruc- } \\
\text { tura aproximada al tipo orgánico }\end{array}$} \\
\hline & & 41 & $45,56 \%$ & 31 & $40,79 \%$ \\
\hline & & 49 & $54,44 \%$ & 45 & $59,21 \%$ \\
\hline \multirow{2}{*}{$\begin{array}{l}\text { Empresas con innovación } \\
\text { radical de proceso }\end{array}$} & No consiguen & 68 & $75,56 \%$ & 52 & $68,42 \%$ \\
\hline & Sí consiguen & 22 & $24,44 \%$ & 24 & $31,58 \%$ \\
\hline \multirow{2}{*}{$\begin{array}{l}\text { Empresas con innovación } \\
\text { radical de producto }\end{array}$} & No consiguen & 56 & $62,22 \%$ & 61 & $80,26 \%$ \\
\hline & Sí consiguen & 34 & $37,78 \%$ & 15 & $19,74 \%$ \\
\hline \multirow{2}{*}{$\begin{array}{l}\text { Empresas con innovación } \\
\text { incremental de producto }\end{array}$} & No consiguen & 57 & $63,33 \%$ & 53 & $69,74 \%$ \\
\hline & Sí consiguen & 33 & $36,67 \%$ & 23 & $30,26 \%$ \\
\hline
\end{tabular}

*p-level<0,05. FUENTE: Elaboración propia.

En consecuencia, la hipótesis $\mathrm{H}_{6}$ se soporta ya que los grupos estratégicos identificados en el colectivo de empresas de la Economía Social que innovan consiguen diferentes tipos de innovación.

\section{5.- Conclusiones e implicaciones}

Este epígrafe presenta las principales conclusiones e implicaciones teóricas y empíricas de la investigación.

En relación a las conclusiones de esta investigación, resaltamos un total de siete, así pues, en primer lugar, las empresas de Economía Social que innovan están menos formalizadas. Apoyan parcialmente este resultado Jansen et al. (2006), quienes concluyen que la formalización constriñe las innovaciones exploratorias, pero mejora las innovaciones de explotación. Por el contrario, Weick (1979) sostiene que la formalización reduce la probabilidad de que los individuos se desvíen del comportamiento estructurado, y como consecuencia, para Zollo y Winter (2002), esto puede ayudar a las unidades a facilitar la replicación y la difusión de la innovación exploratoria.

En segundo lugar, las empresas de Economía Social que innovan presentan niveles más altos de centralización; hecho este que, como ya se indicó previamente, contradice parcialmente lo indicado 
en la literatura, puesto que son numerosos los autores que afirman lo inverso, es decir, las empresas más innovadoras son más descentralizadas.

En tercer lugar, las estructuras organizativas de las empresas de Economía Social se aproximan al tipo orgánico.

En cuarto lugar, las estructuras organizativas de las empresas de Economía Social que innovan presentan diferencias significativas de las que no innovan en sus niveles de formalización (es superior en las empresas de Economía Social que no innovan) y de preparación (es inferior en las empresas de Economía Social que no innovan).

En quinto lugar, las empresas de la Economía Social que innovan se agrupan en dos grupos estratégicos: las empresas que tienen estructuras organizativas próximas al tipo mecanicista y aquellas que poseen estructuras organizativas próximas al tipo orgánico.

En sexto lugar, ninguno de los grupos identificados es isomorfo respecto a las evidencias encontradas en la literatura, puesto que las estructuras orgánicas presentan un nivel bajo de formalización del comportamiento, y también una escasa centralización, hecho este que no es concordante con la literatura consultada. En la misma línea, las estructuras mecanicistas presentan un alto nivel de formalización del comportamiento, pero también una alta centralización, lo que contradice las evidencias localizadas en la literatura. Es decir, los modelos estructurales identificados cumplen los requisitos teóricos asociados con la formalización del comportamiento e incumplen los asociados con la centralización, por tanto, no hallamos modelos estructurales "puros".

En séptimo lugar, las empresas de la Economía Social que innovan se asemejan, consiguen un alto número de innovaciones incrementales de proceso, y se diferencian en que el grupo de empresas de Economía Social con estructuras organizativas próximas al tipo mecanicista genera más innovaciones radicales de producto, es decir, el grupo de empresas de Economía Social que poseen estructuras organizativas próximas al tipo orgánico generan más innovaciones incrementales de proceso.

A modo de síntesis global, la tabla siguiente pone de manifiesto el grado en que se soportan 0 rechazan las hipótesis formuladas. 


\section{Cuadro 2. Síntesis de los resultados relativos a las hipótesis}

\begin{tabular}{|l|c|}
\hline Hipótesis & $\begin{array}{c}\text { Hallazgos } \\
\text { encontrados }\end{array}$ \\
\hline$H_{1}$. La formalización del comportamiento es menor en las empresas de Economía Social que innovan. & Se soporta \\
\hline $\begin{array}{l}\mathrm{H}_{2} \text {. La centralización de la estructura organizativa es mayor en las empresas de Economía Social } \\
\text { que no innovan. }\end{array}$ & Se rechaza \\
\hline $\mathrm{H}_{3}$. La estructura orgánica es más característica de las empresas de Economía Social que innovan. & Se soporta \\
\hline $\begin{array}{l}\mathrm{H}_{4} \text {. La estructura organizativa de las empresas de Economía Social que no innovan difiere de la estructura } \\
\text { organizativa de las empresas de Economía Social que innovan. }\end{array}$ & Se soporta \\
\hline $\begin{array}{l}\mathrm{H}_{5} \text {. Las características o parámetros de diseño de la estructura organizativa revelan la existencia de } \\
\text { diferentes tipos o grupos estratégicos en el colectivo de empresas de Economía Social que innovan. }\end{array}$ & Se soporta \\
\hline $\begin{array}{l}\mathrm{H}_{6} \text {. Los grupos estratégicos identificados en el colectivo de empresas de Economía Social que innovan } \\
\text { generan diferentes tipos de innovación. }\end{array}$ & Se soporta \\
\hline
\end{tabular}

FUENTE: Elaboración propia.

Por otra parte, en lo referente a las implicaciones teóricas, se considera que esta investigación contribuye a subsanar el déficit de conocimiento sobre innovación en las empresas de Economía Social, en particular, en la relación entre la estructura de estas organizaciones y los tipos o resultados de la innovación. Además, el modelo que se emplea en la investigación se justifica, en base a la literatura disponible, es claro, preciso, parsimonioso y puede ser replicado, con las salvedades y las modificaciones que se detallan al final del artículo, en investigaciones futuras.

Por otra parte, la revisión de la literatura sobre los tópicos principales de esta investigación «estructura organizativa", «tipos de innovación" y la aplicación del modelo conceptual propuesto para el sistema de empresas de Economía Social permite concluir el siguiente decálogo de implicaciones empiricas.

En primer lugar, coincidimos con Hassan et al. (2013) en que los directivos, en nuestro caso directivos de empresas de Economía Social, deben enfatizar más la innovación, pues es un instrumento importante para lograr la capacidad competitiva sostenible. Para ello, y en línea con las palabras de Jung, Chow y Wu (2003), deben contar con líderes visionarios que conduzcan la innovación en la empresa.

En segundo lugar, los directivos de las empresas de Economía Social y, más específicamente, los que no innovan deben ser sensibles a la necesidad de contar con estructuras adecuadas que faciliten el desarrollo y el rendimiento de las capacidades de innovación de producto (Menguc y Auh, 2010) y de otros tipos de innovación. 
En tercer lugar, los directivos de las empresas de Economía Social deben ser conscientes que no existe una única estructura organizativa para conseguir diferentes tipos de innovación. Yao-Sheng (2007) y Teece (1996) afirman que se debe elegir la estructura organizativa más adecuada al tipo de innovación que se pretende conseguir, mientras que, Chang y Harrington (2000) consideran que las formas organizativas elegidas deben minimizar el conflicto entre los grupos de interés, si se desea estimular y conseguir distintos tipos de innovación.

En cuarto lugar, los directivos de las empresas de Economía Social deben incorporar a sus estructuras, en la medida que dispongan, recursos, puestos y órganos que estimulen la innovación. En este sentido, Anthony, Johnson, Sinfield y Altman (2008) consideran que sería ideal incorporar a la estructura organizativa los siguientes órganos o puestos vinculados con la innovación: una unidad de formación, que se ocupe de difundir conocimiento, desarrollar habilidades y crear una cultura innovadora (Deal y Kennedy, 1982); un consejo asesor, integrado por directivos de primer nivel, que se ocupe de desarrollar una visión unificada y establecer las prioridades de la innovación; y un comité asesor externo, para mantenerse al día de los avances científicos clave.

En quinto lugar, los directivos de las empresas de Economía Social deben conocer las palabras de Andrew y Sirkin (2008), quienes afirman que las empresas pueden ser innovadoras con cualquier estructura y pueden dejar de serlo con cualquier estructura. Asimismo muestran que las empresas que han tenido un éxito limitado con la innovación están en desacuerdo con la estrategia de negocio 0 tienen un gasto en innovación demasiado bajo o demasiado alto.

En sexto lugar, los directivos de las empresas de Economía Social que no innovan deberían reducir los niveles de formalización del comportamiento de sus estructuras.

En séptimo lugar, los directivos de las empresas de Economía Social que innovan deberían modificar los niveles de centralización en aras de conseguir estructuras organizativas más orgánicas.

En octavo lugar, los directivos de las empresas de Economía Social deben, según Anthony et al. (2008), considerar que contar con una estructura organizativa adecuada les exige fortalecer los esfuerzos de innovación, trabajar para crear herramientas adecuadas, construir un lenguaje de innovación común y asegurarse que las políticas de recursos humanos estén alineadas con la innovación. Asimismo, Lam (2008) considera que es necesario adaptar las formas estructurales a las oportunidades del entorno (tecnológicas y comerciales) e integrar la capacidad de aprendizaje y la creación de conocimiento en los equipos de trabajo.

En noveno lugar, los directivos de las empresas de Economía Social deben ser conscientes que el tiempo es crucial para valorar como la estructura influye en los resultados de la innovación y en los resultados financieros, y que deben esperar resultados inmediatos del proceso innovador (Gunday et al., 2011). 
En décimo lugar, los gobiernos y las administraciones públicas deberían desarrollar políticas públicas que fomenten todos los tipos de innovación en las pymes, y no únicamente las innovaciones radicales (por ejemplo, con subvenciones de l+D) o la entrada en nuevos mercados (con programas que fomenten las exportaciones como prototipo) (Oke et al. 2007).

\section{6.- Limitaciones y líneas futuras de investigación}

A pesar de considerar que está investigación cumple con su finalidad original de aportar valor a los directivos de las empresas de Economía Social y a los académicos interesados en el tema, no está exenta de limitaciones, entre las que se detallan cinco.

La primera limitación se refiere al ámbito temporal y espacial de la investigación, pues la investigación proporciona una visión estática de las empresas de Economía Social en Andalucía. La segunda limitación trata sobre los problemas derivados de la muestra, que aunque es representativa de la población, no excluye problemas de sesgo y heterogeneidad derivados de que quien contesta al cuestionario son los máximos directivos de las entidades o los responsables de la innovación. La tercera limitación alude a los métodos de análisis multivariante empleados en la investigación. La cuarta limitación subraya los problemas de medida del constructo «estructura organizativa» y la consideración, únicamente, de 2 criterios y 4 tipos de innovación. La quinta limitación se refiere al modelo conceptual y, en particular a que únicamente se considera la variable estructura organizativa como determinante de los tipos de innovación en empresas de Economía Social.

Con el objeto de subsanar estas debilidades, se plantean las líneas futuras de investigación que se describen a continuación.

En primer lugar, se proyecta ampliar el ámbito espacial de la investigación a nivel nacional y conseguir de este modo adoptar una perspectiva comparada en línea con la plateada por Bigliardi e Ivo Dormio (2009).

En segundo lugar, y en línea con Keupp et al. (2012), se pretende realizar un estudio longitudinal, con el objeto de observar la evolución de la estructura, de los tipos de innovación y de las relaciones entre ambos.

En tercer lugar, y con el objeto de reducir el sesgo muestral, se desea conseguir una misión multistakeholder de los tópicos objeto de la investigación y, para ello, se obtendrán datos de grupos de interés internos (directivos y operarios) y externos (clientes y proveedores). 
En cuarto lugar, se pretende mejorar el modelo conceptual. Para ello se incluirán más tipos de innovación (innovación comercial e innovación organizativa, al menos) y, además de los resultados de la innovación, se considerará la cadena de valor de la innovación (Hansen y Birkinshaw, 2007); se integran en el modelo variables internas como las competencias de los empleados (Pini y Santangelo, 2005), el apoyo de la alta dirección (Al Shaar et al., 2015), la estructura de propiedad (Kochhar y Parthiban, 1996; Hoskisson, Hitt, Johnson y Grossman, 2002) y la orientación al mercado (Calantone, Harmancioglu y Droge, 2010), y externas como el sector (García y Molero, 2010).

En quinto lugar, y en línea de Walker (2008), poner a prueba la validez de las configuraciones estructurales identificadas en este artículo mediante análisis factorial confirmatorio.

En sexto lugar, incluir, además del factor estructura organizativa, otros factores (internos y externos), que pueden actuar como determinantes de los tipos de innovación y, en último término, de los

resultados económicos, sociales y ambientales de las empresas andaluzas del sector de Economía Social.

\section{Bibliografía}

ACHA, V., GANN, D.M. \& SALTER, A.J. (2005): "Episodic innovation: R\&D strategies for project based environments", Industry \& Innovation, 12(2), 255-281. http://dx.doi.org/10.1080/13662710500087990.

ADAMS, R., BESSANT, J. \& PHELPS, R. (2006): "Innovation management measurement: A review", International Journal of Management Reviews, 8(1), 21-47. http://dx.doi.org/0.1111/j.14682370.2006.00119.x.

AL SHAAR, E.M., KHATTAB, S.A., ALKAIED, R.N. \& MANNA, A.Q. (2015): "The Effect of Top Management Support on Innovation: the Mediating Role of Synergy Between Organizational Structure and Information Technology", International Review of Management and Business Research, 4(2), 499.

AMIT, R. \& ZOTT, C. (2001): "Value creation in e-business", Strategic Management Journal, 22(6-7), 493-520. http://dx.doi.org/10.1002/smj. 187.

ANDERSON, N., DE DREU, C.K. \& NIJSTAD, B.A. (2004): "The routinization of innovation research: A constructively critical review of the state of the science", Journal of Organizational Behavior, 25(2), 147-173. http://dx.doi.org/10.1002/job.236. 
ANDREW, J.P. \& SIRKIN, H.L. (2008): "Aligning for innovation", Global Business and Organizational Excellence, 27(6), 21-39. http://dx.doi.org/10.1002/joe.20230.

ANDRIES, P. \& DEBACKERE, K. (2006): "Adaptation in new technology based ventures: Insights at the company level", International Journal of Management Reviews, 8(2), 91-112. http://dx.doi.org/10.1111/j.1468-2370.2006.00122.x.

ANTHONY, S.D., JOHNSON, M.W., SINFIELD, J.V. \& ALTMAN, E.J. (2008): "Innovation metrics", Harvard Business Review.

http://isites.harvard.edu/fs/docs/icb.topic1179624.files/Innovation\%20Metrics.pdf.

ASCHHOFF, B. \& SCHMIDT, T. (2008): "Empirical evidence on the success of R\&D cooperation-Happy together?", Review of Industrial Organization, 33(1), 41-62. http://dx.doi.org/10.1007/s11151-0089179-7.

ATUAHENE-GIMA, K. \& KO, A. (2001): "An empirical investigation of the effect of market orientation and entrepreneurship orientation alignment on product innovation", Organization Science, 12(1), 54-74. http://dx.doi.org/10.1287/orsc.12.1.54.10121.

BAARD, V. \& WATTS, T. (2007): "Breaking the Paradox of Innovation in Small Businesses through Sustaining and Disruptive Reinvention", Australasian Accounting Business and Finance Journal, 1(2), 3. http://ro.uow.edu.au/aabfj/vol1/iss2/3.

BARZI, F., CORTELEZZI, F., MARSEGUERRA, G. \& ZOIA, M.G. (2015): "Cooperative innovation: In quest of effective partners. Evidence from Italian firms", Innovation, 17(3), 281-307. http://dx.doi.org/10.1080/14479338.2015.1036541.

BENSAOU, M. \& VENKATRAMAN, N. (1995): "Configurations of interorganizational relationships: A comparison between US and Japanese automakers", Management Science, 41(9), 1471-1492. http://dx.doi.org/10.1287/mnsc.41.9.1471.

BIGLIARDI, B. \& IVO DORMIO, A. (2009): "An empirical investigation of innovation determinants in food machinery enterprises", European Journal of Innovation Management, 12(2), 223-242. http://dx.doi.org/10.1108/14601060910953988.

BIRHANU, M.Y., GIRMA, A. \& PUSKUR, R. (2016): "Determinants of success and intensity of livestock feed technologies use in Ethiopia: Evidence from a positive deviance perspective", Technological Forecasting and Social Change, 115(1), 15-25. https://doi.org/10.1016/j.techfore.2016.09.010.

BOER, H. \& DURING, W.E. (2001): "Innovation, what innovation? A comparison between product, process and organisational innovation", International Journal of Technology Management, 22(1-3), 83-107. https://doi.org/10.1504/IJTM.2001.002956.

BOGERS, M. \& WEST, J. (2010): "Contrasting innovation creation and commercialization within open, user and cumulative innovation", User and Cumulative Innovation. Recuperado de http://papers.ssrn.com/sol3/papers.cfm?abstract_id=1751025, http://dx.doi.org/10.2139/ssrn.1751025. 
BRANDYBERRY, A.A. (2003): "Determinants of adoption for organisational innovations approaching saturation", European Journal of Innovation Management, 6(3), 150-158. http://dx.doi.org/10.1108/14601060310486226

BRUNSWICKER, S. \& VANHAVERBEKE, W. (2015): "Open innovation in small and medium sized enterprises (SMEs): External knowledge sourcing strategies and internal organizational facilitators", Journal of Small Business Management, 53(4), 1241-1263. http://dx.doi.org/10.1111/jsbm.12120

BURNS, T.E. \& STALKER, G.M. (1961): "The management of innovation", University of Illinois at Urbana-Champaign's Academy for Entrepreneurial Leadership Historical Research Reference in Entrepreneurship.

CALANTONE, R.J., HARMANCIOGLU, N. \& DROGE, C. (2010): "Inconclusive Innovation «Returns»: A Meta-Analysis of Research on Innovation in New Product Development", Journal of Product Innovation Management, 27(7), 1065-1081. http://dx.doi.org/10.1111/j.1540-5885.2010.00771.x.

CAMISÓN, C. \& BORONAT, M. (2004): "Factores determinantes del desempeño organizativo: efecto industria, incertidumbre y competencias distintivas", Investigaciones Europeas de Dirección y Economía de la Empresa, 10(3), 127-143.

CARDINAL, L.B. (2001): "Technological innovation in the pharmaceutical industry: The use of organizational control in managing research and development", Organization Science, 12(1), 19-36. http://dx.doi.org/10.1287/orsc.12.1.19.10119.

CHANG, M.H. \& HARRINGTON, J.E. (2000): "Centralization vs. decentralization in a multi-unit organization: A computational model of a retail chain as a multi-agent adaptive system", Management Science, 46(11), 1427-1440. http://dx.doi.org/10.1287/mnsc.46.11.1427.12085

CHAUDHRY, T.T. (2013): "Los Clusters y el uso de marcas colectivas en consorcios de exportación", Trimestre Económico, 80(1), 43-76.

CHAVES, R. \& MONZÓN, J.L. (2001): "Economía social y sector no lucrativo. Actualidad científica y perspectivas", CIRIEC-España, Revista de Economía Pública, Social y Cooperativa, 37, 7-23.

CHAVES, R., MONZÓN, J.L. \& ZARAGOZA, G. (2013): "Social Economy: concept, macromagnitudes and sources of employment for the Social Work", Cuadernos de Trabajo Social, 26(1), 19-25.

CLEMENTE, J., DÍAZ, M. \& MARCUELLO, C. (2008): Estudio sobre las cooperativas y sociedades laborales en España, creación de empleo y contribución al desarrollo económico, Ministerio de Trabajo e Inmigración, Código F08348, Universidad de Zaragoza.

CLEMENTE, J., DÍAZ, M., GONZÁLEZ, M.A., MARCUELLO, C. \& NACHAR, P. (2014): "Evolución del comportamiento estratégico de las cooperativas versus empresas capitalistas: una aproximación descriptiva al efecto crisis", REVESCO, Revista de Estudios Cooperativos, 116, 77-102. http://dx.doi.org/10.5209/rev_REVE.2014.v116.45718. 
COHN, S.F. \& TURYN, R.M. (1980): "The structure of the firm and the adoption of process innovations", IEEE transactions on Engineering Management, 4, 98-102. http://dx.doi.org/10.1109/TEM.1980.6447413.

COSH, A., FU, X. \& HUGHES, A. (2012): "Organisation structure and innovation performance in different environments", Small Business Economics, 39(2), 301-317. http://dx.doi.org/10.1007/s11187010-9304-5.

CUEVAS RODRÍGUEZ, G., CABELLO MEDINA, C. \& CARMONA-LAVADO, A. (2014): "Internal and external social capital for radical product innovation: do they always work well together?", British Journal of Management, 25(2), 266-284. http://dx.doi.org/10.1111/1467-8551.12002.

CULEBRAS, Á.L. (2010): La tipología de las empresas innovadoras españolas, Documentos de trabajo IAES-Instituto Universitario de Análisis Económico y Social, Universidad de Alcalá, 8.

DAFT, R.L. (1978): "A dual-core model of organizational innovation", Academy of Management Journal, 21(2), 193-210. http://dx.doi.org/10.2307/255754.

DAFT, R.L. (2012): Organization theory and design, Nelson Education.

DAHLANDER, L. \& GANN, D.M. (2010): "How open is innovation?", Research Policy, 39(6), 699-709. https://doi.org/10.1016/j.respol.2010.01.013.

DAMANPOUR, F. \& ARAVIND, D. (2012): "Managerial Innovation: Conceptions, Processes, and Antecedents", Management and Organization Review, 8 (2), 423-454. https://doi.org/10.1111/j.1740-8784.2011.00233.x.

DAMANPOUR, F. (1991): "Organizational innovation: A meta-analysis of effects of determinants and moderators", Academy of Management Journal, 34(3), 555-590. https://doi.org/10.2307/256406.

DAMANPOUR, F. (1996): "Organizational complexity and innovation: developing and testing multiple contingency models", Management Science, 42(5), 693-716. http://dx.doi.org/10.1287/mnsc.42.5.693.

DAMANPOUR, F. \& GOPALAKRISHNAN, S. (2001): "The dynamics of the adoption of product and process innovations in organizations", Journal of Management Studies, 38(1), 45-65. http://dx.doi.org/10.1111/1467-6486.00227.

DE LUCA, L.M., VERONA, G. \& VICARI, S. (2010): "Market orientation and R\&D effectiveness in High Technology firms: An empirical investigation in the biotechnology industry", Journal of Product Innovation Management, 27(3), 299-320. http://dx.doi.org/10.1111/j.1540-5885.2010.00718.x.

DE MELLO, A.M., MARX, R. \& SALERNO, M. (2012): "Organizational structures to support innovation: how do companies decide?", Revista de Administração e Inovação, 9(4), 05-20. https://doi.org/10.5773/rai.v9i4.623. 
DEAL, T.E. \& KENNEDY, A.A. (1982): "Corporate cultures: The rites and rituals of organizational life", Addison-Wesley, 2, 98-103.

DECANIO, S.J., DIBBLE, C. \& AMIR-ATEFI, K. (2000): "The importance of organizational structure for the adoption of innovations", Management Science, 46(10), 1285-1299. http://dx.doi.org/10.1287/mnsc.46.10.1285.12270.

DEL ÁGUILA, A.R. \& PADILLA, A. (2010): "Factores determinantes de la innovación en empresas de economía social. La importancia de la formación y de la actitud estratégica", CIRIEC-España, Revista de Economía Pública, Social y Cooperativa, 67, 129-155.

DENYER, D. \& NEELY, A. (2004): "Introduction to special issue: innovation and productivity performance in the UK", International Journal of Management Reviews, 5(3 4), 131-135. http://dx.doi.org/10.1111/j.1460-8545.2004.00100.x.

DÍAZ, N.L, AGUIAR, I. \& DE SAÁ, P. (2006): "El conocimiento organizativo tecnológico y la capacidad de innovación: evidencia para la empresa industrial española", Cuadernos de Economía y Dirección de la Empresa, 27, 33-60.

DÍAZ, V. (1998): "Diseño de tipologías de consumidores mediante la utilización conjunta del Análisis Clúster y otras técnicas Multivariantes", Revista Española de Economía Agraria, 182, 75-104.

DOPICO, A., RODRÍGUEZ, R. \& GONZÁLEZ, E. (2012): "La responsabilidad social empresarial y los stakeholders: un análisis clúster", Revista Galega de Economía, 21(1), 1-17.

DRAZIN, R. \& SCHOONHOVEN, C.B. (1996): "Community, population, and organization effects on innovation: A multilevel perspective", Academy of Management Journal, 39(5), 1065-1083. http://dx.doi.org/10.2307/256992.

ELENKOV, D.S. \& MANEV, I.M. (2005): “Top management leadership and influence on innovation: The role of sociocultural context", Journal of Management, 31(3), 381-402. http://dx.doi.org/10.1177/0149206304272151.

FAGERBERG, J. \& VERSPAGEN, B. (2009): "Innovation studies-The emerging structure of a new scientific field", Research Policy, 38(2), 218-233. https://doi.org/10.1016/..respol.2008.12.006.

GARCÍA, A. \& MOLERO, J. (2010): "Factores que afectan a la innovación: dinamismo tecnológico de los sectores y tipo de innovación", Análisis sobre ciencia e innovación en España, 1, 1-24. Recuperado de https://idus.us.es/xmlui/bitstream/handle/11441/41548/factores\%20que\%20afectan.pdf?sequenc $\mathrm{e}=1$ \&isAllowed $=y$

GARCÍA, R. \& CALANTONE, R. (2002): "A critical look at technological innovation typology and innovativeness terminology: a literature review", Journal of Product Innovation Management, 19(2), 110-132. https://doi.org/10.1111/1540-5885.1920110. 
GARCÍA-DEL JUNCO, J., ESPASANDÍN-BUSTELO, F., PALACIOS-FLORENCIO, B. \& DUTSCHKEJEREMIAS, G. (2013): "An approach to the design of a scale for measuring happiness at work Iberian companies", $10^{\text {th }}$ ATINER'S Conference, Athens Institute for Education and Research.

GATIGNON, H. \& XUEREB, J.M. (1997): "Strategic orientation of the firm and new product performance", Journal of Marketing Research, 34, 77-90. https://doi.org/10.2307/3152066.

GEROSKI, P.A. (1995): Innovation and Competitive Advantage, 159, Organisation for Economic Cooperation and Development.

GÓMEZ, J.M. \& ALEXANDRE, G. (2014): "Economía social y comportamiento innovador: estudio empírico de las empresas de economía social en Castilla y Léon". CIRIEC-España, Revista de Economía Pública, Social y Cooperativa, 81, 191-216.

GONZÁLEZ-PERNÍA, J.L. \& PEÑA-LEGAZKUE, I. (2007): "Determinantes de la capacidad de innovación de los negocios emprendedores en España", Economía Industrial, 363, 129-147.

GOPALAKRISHNAN, S. (2000): "Unraveling the links between dimensions of innovation and organizational performance", The Journal of High Technology Management Research, 11(1), 137-153. https://doi.org/10.1016/S1047-8310(00)00024-9.

GUAN, J. \& MA, N. (2003): "Innovative capability and export performance of Chinese firms", Technovation, 23(9), 737-747. https://doi.org/10.1016/S0166-4972(02)00013-5.

GUDMUNDSON, D., TOWER, C.B. \& HARTMAN, E.A. (2003): "Innovation in small businesses: Culture and ownership structure do matter", Journal of Developmental Entrepreneurship, 8(1), 1.

GUNDAY, G., ULUSOY, G., KILICA, K. \& ALPKAN, L. (2011): "Effects of innovation types on firm performance". International Journal of Production Economics, 133(2), 662-676. https://doi.org/10.1016/j.ijpe.2011.05.014.

GUPTA, A.K., TESLUK, P.E. \& TAYLOR, M.S. (2007): "Innovation at and across multiple levels of analysis", Organization Science, 18(6), 885-897. http://dx.doi.org/10.1287/orsc.1070.0337.

HANSEN, M.T. \& BIRKINSHAW, J. (2007): "The innovation value chain", Harvard Business Review, 85(6), 121.

HAO, Q., KASPER, H. \& MUEHLBACHER, J. (2012): "How does organizational structure influence performance through learning and innovation in Austria and China?", Chinese Management Studies, 6(1), 36-52.

http://dx.doi.org/10.1108/17506141211213717

HASSAN, M.U., SHAUKAT, S., NAWAZ, M.S. \& NAZ, S. (2013): "Effects of innovation types on firm performance: an empirical study on Pakistan's manufacturing sector", Pakistan Journal of Commerce and Social Sciences, 7(2), 243-262. 
HERRMANN, P. (2005): "Evolution of strategic management: the need for new dominant designs", International Journal of Management Reviews, 7(2), 111-130. http://dx.doi.org/10.1111/j.14682370.2005.00108.x.

HITT, M.A., KEATS, B.W. \& DEMARIE, S.M. (1998): "Navegando en el nuevo escenario competitivo: La construcción de la flexibilidad estratégica y la ventaja competitiva en el siglo 21", La Academia de Gestión Ejecutiva, 12(4), 22-42.

HITT, M.A. \& IRELAND, R.D. (2000): "The intersection of entrepreneurship and strategic management research", Handbook of Entrepreneurship, 45, 63.

HITT, M.A., HOSKISSON, R.E., JOHNSON, R.A. \& MOESEL, D.D. (1996): "The market for corporate control and firm innovation", Academy of Management Journal, 39(5), 1084-1119. http://dx.doi.org/10.2307/256993.

HOONSOPON, D. \& RUENROM, G. (2012): "The impact of organizational capabilities on the development of radical and incremental product innovation and product innovation performance", Journal of Managerial Issues, 250-276.

HOSKISSON, R.E., HITT, M.A., JOHNSON, R.A. \& GROSSMAN, W. (2002): "Conflicting voices: The effects of institutional ownership heterogeneity and internal governance on corporate innovation strategies", Academy of Management Journal, 45(4), 697-716. http://dx.doi.org/10.2307/3069305.

INE (2000): Encuesta sobre Innovación Tecnológica en las Empresas 1998, Instituto Nacional de Estadística, Madrid.

JANSEN, J.J., VAN DEN BOSCH, F.A. \& VOLBERDA, H.W. (2006): "Exploratory innovation, exploitative innovation, and performance: Effects of organizational antecedents and environmental moderators", Management Science, 52(11), 1661-1674. http://dx.doi.org/10.1287/mnsc.1060.0576.

JOHANNESSEN, J.A., OLSEN, B. \& LUMPKIN, G.T. (2001): "Innovation as newness: what is new, how new, and new to whom?", European Journal of Innovation Management, 4(1), 20-31. http://dx.doi.org/10.1108/14601060110365547.

JUNG, D.I., CHOW, C. \& WU, A. (2003): "The role of transformational leadership in enhancing organizational innovation: Hypotheses and some preliminary findings", The Leadership Quarterly, 14(4), 525-544. https://doi.org/10.1016/S1048-9843(03)00050-X.

KEUPP, M.M., PALMIÉ, M. \& GASSMANN, O. (2012): "The strategic management of innovation: A systematic review and paths for future research", International Journal of Management Reviews, 14(4), 367-390. https://doi.org/10.1111/j.1468-2370.2011.00321.x.

KIM, J. (2015): "A socio-Legal corporate governance model: Analyzing South Korea's social enterprise promotion act using public-Private partnerships", Corporate Ownership and Control, 12(3), 357370 . 
KOCHHAR, R. \& PARTHIBAN, D. (1996): "Institutional investors and firm innovation: A test of competing hypotheses", Strategic Management Journal, 17(1), 73-84.

LAM, A. (2008): "Organizaciones innovadoras: estructura, aprendizaje y adaptación”, Royal Holloway University of London, 1, 163-177.

LAMOTHE, L. \& DUFOUR, Y. (2007): "Systems of interdependency and core orchestrating themes at health care unit level: a configurational approach", Public Management Review, 9(1), 67-85. http://dx.doi.org/10.1080/14719030601181225.

LIU, H. (1995): "Market orientation and firm size: an empirical examination in UK firms", European Journal of Marketing, 29(1), 57-71. http://dx.doi.org/10.1108/03090569510075343.

LÓPEZ, N., MONTES, J.M. \& VÁZQUEZ, C. (2003): "Fuentes tecnológicas para la innovación. Algunos datos para la industria española", Revista Madrid+d, 20.

LÓPEZ-FERNÁNDEZ, M.C., SERRANO-BEDIA, A.M. \& GÓMEZ-LÓPEZ, R. (2011): "Factors encouraging innovation in Spanish hospitality firms", Cornell Hospitality Quarterly, 52(2), 144-152. http://dx.doi.org/10.1177/1938965510393723.

LOVE, J.H. \& ASHCROFT, B. (1999): "Market versus corporate structure in plant-level innovation performance", Small Business Economics, 13(2), 97-109. http://dx.doi.org/10.1023/A:1008182504928.

LUECKE, R. \& KATZ, R. (2003): Harvard business essentials: managing creativity and innovation, Harvard Business School Press.

MACHER, J.T. \& MOWERY, D.C. (2009): "Measuring dynamic capabilities: practices and performance in semiconductor manufacturing", British Journal of Management, 20(1), 41-62. http://dx.doi.org/10.1111/j.1467-8551.2008.00612.x.

MANZINI, E. (2014): "Making things happen: Social innovation and design", Design Issues, 30(1), 5766. http://dx.doi.org/10.1162/DESI_a_00248.

MARTíNEZ, E. (1984): "Aspectos teóricos del Análisis de Clúster y aplicación a la caracterización del electorado. Introducción a las técnicas de Análisis Multivariable", CSIC: Madrid, 165-208.

MATHESON, C. (2009): "Understanding the policy process: The work of Henry Mintzberg", Public Administration Review, 69(6), 1148-1161. https://doi.org/10.1111/j.1540-6210.2009.02072.x.

MCADAM, R. \& KEOGH, W. (2004): "Transitioning towards creativity and innovation measurement in SMEs", Creativity and Innovation Management, 13(2), 126-139. https://doi.org/10.1111/j.09631690.2004.00300.x.

MCMURRAY, A.J. \& WILLIAMS, L. (2004): "Factors impacting on nurse managers' ability to be innovative in a decentralized management structure", Journal of Nursing Management, 12(5), 348-353. https://doi.org/10.1111/j.1365-2834.2004.00426.x. 
MEDINA, M.J., MOZAS, A., BERNAL, E. \& MORAL, E. (2014): "Factores determinantes para la exportación en las empresas cooperativas oleícolas andaluzas", CIRIEC-España, Revista de Economía Pública, Social y Cooperativa, 81, 241-262.

MENGUC, B. \& AUH, S. (2010): "Development and return on execution of product innovation capabilities: The role of organizational structure", Industrial Marketing Management, 39(5), 820-831. https://doi.org/10.1016/j.indmarman.2009.08.004.

MILES, R.E., SNOW, C.C., MEYER, A.D. \& COLEMAN, H.J. (1978): "Organizational strategy, structure, and process", Academy of Management Review, 3(3), 546-562. https://doi.org/10.5465/AMR.1978.4305755.

MINTZBERG, H. (1996): "Managing government, governing management", Harvard Business Review, 74(3), 75 .

MINTZBERG, H. (2012): La Estructuración de las organizaciones, Ariel.

MONE, M.A., MCKINLEY, W. \& BARKER, V.L. (1998): "Organizational decline and innovation: A contingency framework", Academy of Management Review, 23(1), 115-132. https://doi.org/10.5465/AMR.1998.192965.

MORALES, A.C. \& GARCÍA, J. (2007): "Innovación empresarial y economía social: un estudio empírico a nivel andaluz". Strengthening and Building Communities: The Social Economy in a Changing

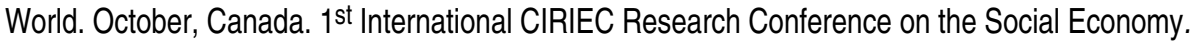

MOSEY, S. (2005): "Understanding new to market product development in SMEs", International Journal of Operations \& Production Management, 25, 2, 11430.

http://dx.doi.org/10.1108/01443570510576994.

MOSQUET, X., RUSSO, M., WAGNER, K., ZABLIT, H. \& ARORA, A. (2014): "Accelerating Innovation: New Challenges for Automakers", The Boston Consulting Group.

MYERS, S. \& MARQUIS, D.G. (1969): "Successful industrial innovations. A study of factors underlying innovation in selected firms".

NAG, R., CORLEY, K.G. \& GIOIA, D.A. (2007): "The intersection of organizational identity, knowledge, and practice: Attempting strategic change via knowledge grafting", Academy of Management Journal, 50(4), 821-847. http://dx.doi.org/10.5465/AMJ.2007.26279173.

NAHM, A.Y., VONDEREMBSE, M.A. \& KOUFTEROS, X. (2003): "The impact of organizational structure on time-based manufacturing and plant performance", Journal of Operations Management, 21, 281-306. https://doi.org/10.1016/S0272-6963(02)00107-9.

NONAKA, I. \& TOYAMA, R. (2003): "The knowledge-creating theory revisited: knowledge creation as a synthesizing process", Knowledge Management Research \& Practice, 1(1), 2-10. https://doi.org/10.1057/palgrave.kmrp.8500001. 
NORD, W.R. \& TUCKER, S. (1987): Implementing routine and radical innovations, Free Press.

OCDE, O.M. (2005): The measurement of scientific and technological activities, Proposes Guidelines for Collecting and Interpreting Innovation Data, Paris.

OKE, A., BURKE, G. \& MYERS, A. (2007): "Innovation types and performance in growing UK SMEs", International Journal of Operations \& Production Management, 27(7), 735-753. http://dx.doi.org/10.1108/01443570710756974.

O'NEILL, H.M., POUDER, R.W. \& BUCHHOLTZ, A.K. (1998): "Patterns in the diffusion of strategies across organizations: Insights from the innovation diffusion literature", Academy of Management Review, 23(1), 98-114. http://dx.doi.org/10.5465/AMR.1998.192963.

PAGE, A.L. \& SCHIRR, G.R. (2008): "Growth and development of a body of knowledge: 16 years of new product development research, 1989-2004", Journal of Product Innovation Management, 25(3), 233-248. http://dx.doi.org/10.1111/j.1540-5885.2008.00297.x.

PALMER, J.C. \& WRIGHT, R.E. (2010): "Product innovation in small firms: An empirical assessment", The Journal of Applied Business and Economics, 11(3), 33.

PÉREZ, M.C. \& VALIENTE, L. (2015): "Impacto territorial del autoempleo en la economía social en España”, CIRIEC-España, Revista de Economía Pública, Social y Cooperativa, 83, 83-114.

PIENING, E.P. \& SALGE, T.O. (2015): "Understanding the antecedents, contingencies, and performance implications of process innovation: A dynamic capabilities perspective", Journal of Product Innovation Management, 32(1), 80-97. http://dx.doi.org/10.1111/jpim.12225.

PINI, P. \& SANTANGELO, G.D. (2005): "Innovation types and labour organizational practices: a comparison of foreign and domestic firms in the Reggio Emilia industrial districts", Economics of Innovation and New Technology, 14(4), 251-276. http://dx.doi.org/10.1080/1043859042000269089.

PITTAWAY, L., ROBERTSON, M., MUNIR, K., DENYER, D. \& NEELY, A. (2004): "Networking and innovation: a systematic review of the evidence", International Journal of Management Reviews, 5(3 4), 137-168. http://dx.doi.org/10.1111/j.1460-8545.2004.00101.x.

QUADROS, R., FURTADO, A., BERNARDES, R. \& FRANCO, E. (2001): "Technological innovation in Brazilian industry: an assessment based on the São Paulo innovation survey", Technological Forecasting and Social Change, 67(2), 203-219. https://doi.org/10.1016/S0040-1625(00)00123-2.

RODRÍGUEZ, M.J. \& GUZMÁN, C. (2013): "Innovation in social economy firms", Management Decision, 51(5), 986-998. http://dx.doi.org/10.1108/MD-08-2012-0538.

SHAPIRO, C. (2001): "Navigating the patent thicket: Cross licenses, patent pools, and standard setting", Innovation Policy and the Economy, 1, 119-150. https://doi.org/10.1086/ipe.1.25056143.

SORENSEN, J.B. \& STUART, T.E. (2000): "Aging, obsolescence, and organizational innovation", Administrative Science Quarterly, 45(1), 81-112. http://dx.doi.org/10.2307/2666980. 
STORY, V.M., DANIELS, K., ZOLKIEWSKI, J. \& DAINTY, A.R. (2014): "The barriers and consequences of radical innovations: Introduction to the issue", Industrial Marketing Management, 43(8), 12711277. https://doi.org/10.1016/j.indmarman.2014.09.001.

SUBRAMANIAN, A., \& NILAKANTA, S. (1996): "Organizational innovativeness: exploring the relationship between organizational determinants of innovation, types of innovations, and measures of organizational performance", Omega, 24(6), 631-647. https://doi.org/10.1016/S03050483(96)00031-X.

TEECE, D.J. (1996): "Firm organization, industrial structure, and technological innovation", Journal of Economic Behavior \& Organization, 31(2), 193-224. https://doi.org/10.1016/S0167-2681(96)00895-5.

TEECE, D.J. (2016): "Dynamic capabilities and entrepreneurial management in large organizations: Toward a theory of the (entrepreneurial) firm", European Economic Review, 86, 202-216. https://doi.org/10.1016/j.euroecorev.2015.11.006.

TIDD, J. (2001): "Innovation management in context: environment, organization and performance", International Journal of Management Reviews, 3(3), 169-183. https://doi.org/10.1111/14682370.00062.

TUSHRNAN, M.L., ANDERSON, P.C. \& O'REIUY, C. (1997): "Technology cycles, innovation streams, and ambidextrous organizations: organization renewal through innovation streams and strategic change", Managing Strategic Innovation and Change, 3, 23.

WALKER, R.M. (2008): "An empirical evaluation of innovation types and organizational and environmental characteristics: Towards a configuration framework", Journal of Public Administration Research and Theory, 18(4), 591-615. https://doi.org/10.1093/jopart/mum026.

WANG, C.L. (2008): "Entrepreneurial orientation, learning orientation, and firm performance", Entrepreneurship Theory and Practice, 32(4), 635-657. https://doi.org/10.1111/j.15406520.2008.00246.x.

WEICK, K.E. (1979): "Cognitive processes in organizations", Research in Organizational Behavior, 1(1), 41-74.

WEST, M.A. \& FARR, J.L. (1989): "Innovation at work: Psychological perspectives", Social Behaviour, $4(1), 15-30$.

WU, W.Z. \& ZHUANG, Z.M. (2003): "The Layout and Innovation of Tourism Products in Experience Economy Era-A Case Study on Experience-Exploitation of Ancient Village Tourism Product", Tourism Tribune, 6.

YAO-SHENG, L. (2007): "The effects of knowledge management strategy and organization structure on innovation", International Journal of Management, 24(1), 53. 
ZAHRA, S.A. \& BOGNER, W.C. (2000): "Technology strategy and software new ventures' performance: Exploring the moderating effect of the competitive environment", Journal of Business Venturing, 15(2), 135-173. https://doi.org/10.1016/S0883-9026(98)00009-3.

ZHENG, W. (2010): "A social capital perspective of innovation from individuals to nations: where is empirical literature directing us?", International Journal of Management Reviews, 12(2), 151-183. https://doi.org/10.1111/j.1468-2370.2008.00247.x.

ZOLLO, M. \& WINTER, S.G. (2002): "Deliberate learning and the evolution of dynamic capabilities", Organization Science, 13(3), 339-351. http://dx.doi.org/10.1287/orsc.13.3.339.2780.

ZORTEA-JOHNSTON, E., DARROCH, J. \& MATEAR, S. (2012): "Business orientations and innovation in small and medium sized enterprises", International Entrepreneurship and Management Journal, 8(2), 145-164. http://dx.doi.org/10.1007/s11365-011-0170-7. 